TRANSACTIONS OF THE

AMERICAN MATHEMATICAL SOCIETY

Volume 358, Number 9, September 2006, Pages 3851-3881

S 0002-9947(05)03761-X

Article electronically published on July 26, 2005

\title{
ON THE EIGENVALUE PROBLEM FOR PERTURBED NONLINEAR MAXIMAL MONOTONE OPERATORS IN REFLEXIVE BANACH SPACES
}

\author{
ATHANASSIOS G. KARTSATOS AND IGOR V. SKRYPNIK
}

\begin{abstract}
Let $X$ be a real reflexive Banach space with dual $X^{*}$ and $G \subset X$ open and bounded and such that $0 \in G$. Let $T: X \supset D(T) \rightarrow 2^{X^{*}}$ be maximal monotone with $0 \in D(T)$ and $0 \in T(0)$, and $C: X \supset D(C) \rightarrow X^{*}$ with $0 \in D(C)$ and $C(0) \neq 0$. A general and more unified eigenvalue theory is developed for the pair of operators $(T, C)$. Further conditions are given for the existence of a pair $(\lambda, x) \in(0, \infty) \times(D(T+C) \cap \partial G)$ such that

(**) $\quad T x+\lambda C x \ni 0$.

The "implicit" eigenvalue problem, with $C(\lambda, x)$ in place of $\lambda C x$, is also considered. The existence of continuous branches of eigenvectors of infinite length is investigated, and a Fredholm alternative in the spirit of Necas is given for a pair of homogeneous operators $T, C$. No compactness assumptions have been made in most of the results. The degree theories of Browder and Skrypnik are used, as well as the degree theories of the authors involving densely defined perturbations of maximal monotone operators. Applications to nonlinear partial differential equations are included.
\end{abstract}

\section{INTRODUCTION-PRELIMINARIES}

Unless otherwise stated, the symbol $X$ stands for a real reflexive Banach space which has been renormed so that it and its dual $X^{*}$ are locally uniformly convex. The symbol $\|\cdot\|$ stands for the norm of $X, X^{*}$ and $J: X \rightarrow X^{*}$ is the normalized duality mapping. In what follows, "continuous" means "strongly continuous" and the symbol " $\rightarrow$ " ("- $\rightarrow$ ") means strong (weak) convergence.

The symbol $\mathcal{R}\left(\mathcal{R}_{+}\right)$stands for the set $(-\infty, \infty)([0, \infty))$ and the symbols $\partial D, \bar{D}$ denote the strong boundary and closure of the set $D$, respectively. We denote by $B_{r}(0)$ the open ball of $X$ or $X^{*}$ with center at zero and radius $r>0$.

For an operator $T: X \rightarrow 2^{X^{*}}$ we denote by $D(T)$ the effective domain of $T$, i.e. $D(T)=\{x \in X: T x \neq \emptyset\}$. We denote by $G(T)$ the graph of $T$, i.e. $G(T)=\{(x, y): x \in D(T), y \in T x\}$. An operator $T: X \supset D(T) \rightarrow 2^{X^{*}}$ is called "monotone" if for every $x, y \in D(T)$ and every $u \in T x, v \in T y$ we have

$$
\langle u-v, x-y\rangle \geq 0 .
$$

A monotone operator $T$ is "maximal monotone" if $G(T)$ is maximal in $X \times X^{*}$, when $X \times X^{*}$ is partially ordered by inclusion. In our setting, a monotone operator

Received by the editors May 6, 2003 and, in revised form, June 3, 2004.

2000 Mathematics Subject Classification. Primary 47H14, 47H07, 47H11.

Key words and phrases. Maximal monotone operators, $\left(S_{+}\right)$-mappings, Browder's degree, Skrypnik's degree, degree for sums of densely defined mappings, nonlinear eigenvalue problems.

(C)2005 American Mathematical Society Reverts to public domain 28 years from publication 3851 
$T$ is maximal if and only if $R(T+\lambda J)=X^{*}$ for all $\lambda \in(0, \infty)$. If $T$ is maximal monotone, then the operator $T_{t} \equiv\left(T^{-1}+t J^{-1}\right)^{-1}: X \rightarrow X^{*}$ is bounded, demicontinuous, maximal monotone and such that $T_{t} x \rightarrow T^{\{0\}} x$ as $t \rightarrow 0^{+}$for every $x \in D(T)$, where $T^{\{0\}} x$ denotes the element $y^{*} \in T x$ of minimum norm, i.e. $\left\|T^{\{0\}} x\right\|=\inf \left\{\left\|y^{*}\right\|: y^{*} \in T x\right\}$. In our setting, this infimum is always attained and $D\left(T^{\{0\}}\right)=D(T)$. Also, $T_{t} x \in T J_{t} x$, where $J_{t} \equiv I-t J^{-1} T_{t}: X \rightarrow X$ and satisfies $\lim _{t \rightarrow 0} J_{t} x=x$ for all $x \in \overline{\operatorname{co} D(T)}$, where $\operatorname{co} A$ denotes the convex hull of the set $A$. In addition, $x \in D(T)$ and $t_{0}>0$ imply $\lim _{t \rightarrow t_{0}} T_{t} x=T_{t_{0}} x$. The operators $T_{t}, J_{t}$ were introduced by Brézis, Crandall and Pazy in 2]. For their basic properties, we refer the reader to [2] as well as Pascali and Sburlan [18, pp. 128-130]. In our setting, the duality mapping $J$ is single-valued and bicontinuous.

An operator $T: X \supset D(T) \rightarrow Y$, with $Y$ another real Banach space, is "bounded" if it maps bounded subsets of $D(T)$ onto bounded sets. It is "compact" if it is continuous and maps bounded subsets of $D(T)$ onto relatively compact subsets of $Y$. It is "demicontinuous" ("completely continuous") if it is strong-weak (weak-strong) continuous on $D(T)$.

Given an operator $T: X \supset D(T) \rightarrow 2^{X^{*}}$, we say that $T$ has the property $\mathcal{P}$ "locally" on $G \subset X$ if for every $x_{0} \in D(T) \cap G$ there exists a closed ball $\overline{B_{r}\left(x_{0}\right)} \subset G$ such that $T$ has the property $\mathcal{P}$ on $D(T) \cap \overline{B_{r}\left(x_{0}\right)}$. If $G=X$, then we simply say that $T$ has "locally" the property $\mathcal{P}$.

We say that an operator $T: X \supset D(T) \rightarrow 2^{X^{*}}$ satisfies condition " $(S)$ " on $B \subset D(T)$ if $\left\{x_{n}\right\} \subset B, x_{n} \rightarrow x_{0}$ and

$$
\lim _{n \rightarrow \infty}\left\langle u_{n}, x_{n}-x_{0}\right\rangle=0,
$$

for some $u_{n} \in T x_{n}$, imply $x_{n} \rightarrow x_{0}$.

We say that an operator $T: X \supset D(T) \rightarrow 2^{X^{*}}$ satisfies condition " $\left(S_{+}\right)$" on $B \subset D(T)$ if $\left\{x_{n}\right\} \subset B, x_{n} \rightarrow x_{0}$ and

$$
\limsup _{n \rightarrow \infty}\left\langle u_{n}, x_{n}-x_{0}\right\rangle \leq 0
$$

for some $u_{n} \in T x_{n}$, imply $x_{n} \rightarrow x_{0}$.

Let $L$ be a dense subspace of $X$. An operator $T: X \supset D(T) \rightarrow X^{*}$, with $L \subset D(T)$, is said to satisfy condition $\left(\widetilde{S}_{+}\right)_{L}$ if $\left\{u_{n}\right\} \subset L, u_{n} \rightarrow u_{0}, T u_{n} \rightarrow h_{0}^{*}$ and

$$
\limsup _{n \rightarrow \infty}\left\langle T u_{n}, u_{n}-u_{0}\right\rangle \leq 0
$$

imply $u_{n} \rightarrow u_{0}, u_{0} \in D(T)$ and $T u_{0}=h_{0}^{*}$.

An operator $T: X \supset D(T) \rightarrow X^{*}$ with $L \subset D(T)$ is said to satisfy condition $\left(\widetilde{S}_{+}\right)$if $\left\{u_{n}\right\} \subset D(T), u_{n} \rightarrow u_{0}, T u_{n} \rightarrow h_{0}^{*}$ and

$$
\limsup _{n \rightarrow \infty}\left\langle T u_{n}, u_{n}-u_{0}\right\rangle \leq 0
$$

imply $u_{n} \rightarrow u_{0}, u_{0} \in D(T)$ and $T u_{0}=h_{0}^{*}$.

We say that the operator $T: X \supset D(T) \rightarrow 2^{X^{*}}$ satisfies condition $\left(S_{q}\right)$ on a set $A \subset D(T)$ if for every sequence $\left\{x_{n}\right\} \subset A$ such that $x_{n} \rightarrow x_{0} \in X$ and any $y_{n}^{*} \in T x_{n}$, with $y_{n}^{*} \rightarrow$ (some) $y^{*} \in X^{*}$, we have $x_{n} \rightarrow x_{0}$. If $A=D(T)$, then we say that $T$ satisfies $\left(S_{q}\right)$.

Obviously, if an operator is of type $(S)$ on $A \subset D(T)$, then it is also of type $\left(S_{q}\right)$ on $A$. The following lemma can be found in Zeidler [24, p. 915]. 
Lemma A. Let $T: X \supset D(T) \rightarrow 2^{X^{*}}$ be maximal monotone. Then the following are true:

(i) $\left\{x_{n}\right\} \subset D(T), x_{n} \rightarrow x_{0}$ and $T x_{n} \ni y_{n} \rightarrow y_{0}$ imply $x_{0} \in D(T)$ and $y_{0} \in T x_{0}$.

(ii) $\left\{x_{n}\right\} \subset D(T), x_{n} \rightarrow x_{0}$ and $T x_{n} \ni y_{n} \rightarrow y_{0}$ imply $x_{0} \in D(T)$ and $y_{0} \in T x_{0}$.

From Lemma A we see that either one of (i) or (ii) implies that the graph $G(T)$ of the operator $T$ is closed, i.e. $G(T) \equiv\{(u, x) ; x \in D(T), u \in T x\}$ is a closed subset of $X \times X^{*}$.

Unless otherwise stated, the symbol $d(T, G, p)$ denotes the Leray-Schauder degree of the mapping $T$ on the closed and bounded set $G$ w.r.t. $p \notin T(\partial G)$.

For facts involving monotone operators, and other related concepts, the reader is referred to Barbu [1], Browder [3], Cioranescu [7, Pascali and Sburlan [18, Simons 21], Skrypnik [23], and Zeidler [24]. We cite the books of Browder [3], Lloyd [17, Petryshyn [19, Rothe [20, Skrypnik 23] and the papers of Browder [4]-6] and Kartsatos and Skrypnik [13] as references to degree theories.

For recent nonlinear eigenvalue results we refer the reader to Guan and Kartsatos [9, Kartsatos [11, the authors [12, Li and Huang [16, and Skrypnik [23. p. 124]. For a recent "invariance of domain" theory, we cite the paper of the authors [14.

It is our main intention here to establish an eigenvalue theory for various classes of operators $T+C$, with $T$ maximal monotone, acting from the space $X$ to its dual $X^{*}$. Guan and Kartsatos gave a series of results in [9] involving the existence of eigenvalues and eigenvectors of inclusions of the type

$$
T x+\lambda C x \ni 0
$$

containing maximal monotone or $m$-accretive operators $T$ and perturbing operators $C$. Roughly speaking, these results use or imply conditions of the type $\|C x\| \geq$ $\alpha, x \in \partial G$, where $G$ is an open and bounded subset of $X$. The reason for such conditions is that when they hold they guarantee that the degree of a certain mapping associated with problem $(*)$ is zero (cf., e.g., Guan and Kartsatos 9, Proof of Theorem A]). These considerations were substantially improved by the authors in [12]. In fact, we considered in [12] implicit eigenvalue problems of the type

$$
T x+C(\lambda, x)=0,
$$

for many combinations of operators $T, C$ with $T m$-accretive, or maximal monotone. Our results in [12] were based on various compactness assumptions on the operator $C$ or the resolvents of the operator $T$. We also showed in [12] that one can even obtain normalized eigenvectors $x$ for such problems, which are lying on the boundaries of sets which may be unbounded in the norm of the underlying energy space.

This paper can be considered to be a continuation of all the above mentioned eigenvalue papers. In particular, we assume here that problems like

$$
T x+\lambda C x+\varepsilon J x \ni 0
$$

have no solutions in $D(T) \cap G$ for some $\lambda>0$ and all small $\varepsilon>0$.

In Section 1 we give a result that guarantees the existence of eigenvalues for problems of type $(* *)$

$$
T x+C(\lambda, x) \ni 0
$$


where $T$ is maximal monotone and $C(\lambda, x)$ is demicontinuous, bounded and of type $\left(S_{+}\right)$.

In Section 2 we use Browder's degree theory in [6] involving multi-valued maximal monotone operators $T$ and demicontinuous operators $C$ defined on the closures of bounded open sets in $X$.

In Section 3 we first consider the problem

$$
T x+\lambda C x=0
$$

for single-valued densely defined operators $T, C$. Our approach here uses the degree theory that was developed by the authors in [13].

Section 4 is devoted to the existence of eigenvalues for operators $T, C$, where $T$ is maximal monotone and $C$ is densely defined. Here, we use the new degree theory developed by the authors in [15].

In Section 5 we give a Fredholm alternative result in the spirit of Necas 8 , p. 61] concerning the surjectivity of operators $\lambda T+C$ whenever $\lambda(\geq 1)$ is not an eigenvalue for the pair $(T, C)$. In this result both operators $T, C$ are positively homogeneous of degree $\gamma \geq 1$.

Section 6 is devoted to continuous and bounded operators $C$ defined on $\overline{D(T)}$, and maximal monotone operators $T$ with compact resolvents.

In Section 7 we demonstrate the fact that our eigenvalue results can give rise to the existence of continuous branches of eigenvectors of infinite length.

Applications to partial differential equations are given in Section 8.

\section{Demicontinuous operators $C$ of type $\left(S_{+}\right)$}

Our main purpose in this section is to prove Theorem 1 below about the implicit eigenvalue problem

$$
T x+C(\lambda, x) \ni 0 .
$$

Let $G \subset X$ be open and bounded, $\Lambda>0$. An operator $C:[0, \Lambda] \times \bar{G} \rightarrow X^{*}$ is "demicontinuous" if $[0, \Lambda] \times \bar{G} \ni\left(t_{n}, x_{n}\right) \rightarrow\left(t_{0}, x_{0}\right)$ implies $C\left(t_{n}, x_{n}\right) \rightarrow C\left(t_{0}, x_{0}\right)$. A demicontinuous operator $C(t, x)$ as above is continuous in $t$ "uniformly w.r.t. $x \in \bar{G}$ " if $[0, \Lambda] \ni t_{n} \rightarrow t_{0}$ implies $C\left(t_{n}, x\right) \rightarrow C\left(t_{0}, x\right)$ uniformly w.r.t. $x \in \bar{G}$. A demicontinuous operator $C$ as above is said to satisfy condition " $\left(S_{+}\right)$" if for every $\lambda \in(0, \Lambda]$ and every sequence $\left\{x_{n}\right\} \subset \bar{G}$ with $x_{n} \rightarrow x_{0}$ and

$$
\limsup _{n \rightarrow \infty}\left\langle C\left(\lambda, x_{n}\right), x_{n}-x_{0}\right\rangle \leq 0
$$

we have $x_{n} \rightarrow x_{0}$.

Theorem 1. Let $G \subset X$ be open and bounded. Let $T: X \supset D(T) \rightarrow 2^{X^{*}}$ be maximal monotone with $0 \in D(T) \cap G$ and $0 \in T(0)$. Let $C:[0, \Lambda] \times \bar{G} \rightarrow X^{*}$ be demicontinuous, bounded, of type $\left(S_{+}\right)$, and such that $C(0, x)=0, x \in \bar{G}$, and $C(t, x)$ is continuous in $t$ uniformly w.r.t. $x \in \bar{G}$. Let $\varepsilon, \varepsilon_{0}$ be positive numbers. Assume that

$(\mathcal{P})$ there exists $\lambda \in(0, \Lambda]$ such that the inclusion

$$
T x+C(\lambda, x)+\varepsilon J x \ni 0
$$

has no solution $x \in D(T) \cap G$. Then

(i) there exists $\left(\lambda_{0}, x_{0}\right) \in(0, \Lambda] \times(D(T) \cap \partial G)$ such that

$$
T x_{0}+C\left(\lambda_{0}, x_{0}\right)+\varepsilon J x_{0} \ni 0 ;
$$


(ii) if $0 \notin T(D(T) \cap \partial G)$, $T$ satisfies condition $\left(S_{q}\right)$ on $\partial G$, and property $(\mathcal{P})$ is satisfied for every $\varepsilon \in\left(0, \varepsilon_{0}\right]$, then there exists $\left(\lambda_{0}, x_{0}\right) \in(0, \Lambda] \times(D(T) \cap \partial G)$ such that $T x_{0}+C\left(\lambda_{0}, x_{0}\right) \ni 0$.

Theorem 1 was proved by Li and Huang [16. Theorem 3.1] under the assumption that $C(\lambda, x) \equiv \lambda C x$ and $C$ is a compact operator. We should note here that these authors should have assumed that $0 \in T(0)$. In fact, their proof is based on the homotopy invariance of the Leray-Schauder degree for the homotopy function $H \equiv$ $I-\left(T_{s}+\varepsilon J\right)^{-1}(-\lambda C)$ on $\bar{G}$. However, this function $H$ is not generally homotopic to the identity $I$ for $\lambda=0$, a fact that was used in [15]. It is homotopic to $I$ if we assume that $0 \in T(0)$. Properties like $(\mathcal{P})$ were assumed by Guan and Kartsatos in 9.

Proof of Theorem 1. (i) Assume that (2) is not true. Then for every $\lambda \in(0, \Lambda]$ the equation

$$
T x+C(\lambda, x)+\varepsilon J x \ni 0
$$

has no solution $x \in D(T) \cap \partial G$. We note that this is also true for $\lambda=0$ because $(T+\varepsilon J) G \ni 0$ and the operator $T+\varepsilon J$ is injective by the strict monotonicity of the duality mapping. We set $H(\lambda, x) \equiv T x+C(\lambda, x)+\varepsilon J x$ and observe that

$$
H(\lambda, D(T) \cap \partial G) \not \supset 0, \quad \lambda \in[0, \Lambda] .
$$

We are now going to show that there exist $s_{0}>0, \lambda_{0} \in(0, \Lambda]$ such that for every $s \in\left(0, s_{0}\right], \lambda \in\left(0, \lambda_{0}\right]$ we have $0 \notin H_{1}(s, \lambda, \partial G)$, where

$$
H_{1}(s, \lambda, x) \equiv T_{s} x+C(\lambda, x)+\varepsilon J x .
$$

Assume that this is not true. Then there exist $s_{n} \downarrow 0, \lambda_{n} \downarrow 0, x_{n} \in \partial G$ with $x_{n} \rightarrow x_{0}, J x_{n} \rightarrow j^{*}$, for some $x_{0} \in X$ and $j^{*} \in X^{*}$, and such that

$$
T_{s_{n}} x_{n}+C\left(\lambda_{n}, x_{n}\right)+\varepsilon J x_{n}=0 .
$$

This implies

$$
\begin{aligned}
\left\langle T_{s_{n}} x_{n}, x_{n}-x_{0}\right\rangle= & -\left\langle C\left(\lambda_{n}, x_{n}\right), x_{n}-x_{0}\right\rangle-\varepsilon\left\langle J x_{n}, x_{n}-x_{0}\right\rangle \\
\leq & \left\|C\left(\lambda_{n}, x_{n}\right)\right\|\left\|x_{n}-x_{0}\right\|-\varepsilon\left\langle J x_{n}-J x_{0}, x_{n}-x_{0}\right\rangle \\
& -\varepsilon\left\langle J x_{0}, x_{n}-x_{0}\right\rangle \\
\leq & \left\|C\left(\lambda_{n}, x_{n}\right)\right\|\left\|x_{n}-x_{0}\right\|-\varepsilon\left\langle J x_{0}, x_{n}-x_{0}\right\rangle .
\end{aligned}
$$

Thus,

$$
\limsup _{n \rightarrow \infty}\left\langle T_{s_{n}} x_{n}, x_{n}-x_{0}\right\rangle \leq \lim _{n \rightarrow \infty}\left[\left\|C\left(\lambda_{n}, x_{n}\right)\right\|\left\|x_{n}-x_{0}\right\|\right]-\varepsilon \lim _{n \rightarrow \infty}\left\langle J x_{0}, x_{n}-x_{0}\right\rangle=0 .
$$

Here, we have used the fact that $\left|\left\|C\left(\lambda_{n}, x_{n}\right)\right\|-\left\|C\left(0, x_{n}\right)\right\|\right|=\left\|C\left(\lambda_{n}, x_{n}\right)\right\| \rightarrow 0$ by the continuity of $C(t, x)$ in $t$ which is uniform w.r.t. $x \in \bar{G}$. Also, (5) implies

$$
T_{s_{n}} x_{n} \rightarrow-\varepsilon j^{*},
$$

which in turn gives

$$
\limsup _{n \rightarrow \infty}\left\langle T_{s_{n}} x_{n}, x_{n}\right\rangle \leq\left\langle-\varepsilon j^{*}, x_{0}\right\rangle .
$$

Now, fix $x \in D(T), x^{*} \in T x$. Then, as in Browder [4, Proof of Theorem 12],

$$
\begin{aligned}
\liminf _{n \rightarrow \infty}\left\langle T_{s_{n}} x_{n}, x_{n}\right\rangle & \geq \liminf _{n \rightarrow \infty}\left\langle T_{s_{n}} x_{n}, x\right\rangle+\left\langle x^{*}, x_{0}-x\right\rangle \\
& =\left\langle-\varepsilon j^{*}, x\right\rangle+\left\langle x^{*}, x_{0}-x\right\rangle .
\end{aligned}
$$


Combining (8) and (9) we get

$$
\left\langle-\epsilon j^{*}-x^{*}, x_{0}-x\right\rangle \geq 0,
$$

which, by the maximal monotonicity of $T$, implies $x_{0} \in D(T)$. Letting $x=x_{0}$ in (9) and using (8) we obtain

$$
\lim _{n \rightarrow \infty}\left\langle T_{s_{n}} x_{n}, x_{n}\right\rangle=\lim _{n \rightarrow \infty}\left\langle T_{s_{n}} x_{n}, x_{0}\right\rangle=\left\langle-\varepsilon j^{*}, x_{0}\right\rangle .
$$

This implies

$$
\lim _{n \rightarrow \infty}\left\langle T_{s_{n}} x_{n}, x_{n}-x_{0}\right\rangle=0 .
$$

Using this with (5) we get

$$
\varepsilon\left\langle J x_{n}, x_{n}-x_{0}\right\rangle=-\left\langle T_{s_{n}} x_{n}, x_{n}-x_{0}\right\rangle-\left\langle C\left(\lambda_{n}, x_{n}\right), x_{n}-x_{0}\right\rangle,
$$

which gives

$$
\lim _{n \rightarrow \infty}\left\langle J x_{n}, x_{n}-x_{0}\right\rangle=0
$$

Since the duality mapping $J$ is of type $(S)$, we have $x_{n} \rightarrow x_{0} \in \partial G, J x_{n} \rightarrow$ $J x_{0}=j^{*}$. Now, $J_{s_{n}} x_{n}=x_{n}-s_{n} J^{-1} T_{s_{n}} x_{n} \rightarrow x_{0}$. Here, we have used the fact that $\left\{T_{s_{n}} x_{n}\right\}$ and $J^{-1}$ are bounded and $s_{n} \downarrow 0$. Consequently, $T_{s_{n}} x_{n} \in T J_{s_{n}} x_{n}$, $J_{s_{n}} x_{n} \rightarrow x_{0}, T_{s_{n}} x_{n} \rightarrow-\varepsilon j^{*}=-\varepsilon J x_{0}$ and the closedness of $T$ (see Lemma A) imply that $T x_{0}+\varepsilon J x_{0} \ni 0$. However, $x_{0} \in D(T) \cap \partial G$ is a contradiction because we already have $0 \in(T+\varepsilon J) G$, and the operator $T+\varepsilon J$ is injective by the strict monotonicity of the duality mapping. Thus, our assertion is true.

Now, we fix $s \in\left(0, s_{0}\right], \lambda \in\left(0, \lambda_{0}\right]$ and consider the homotopy function

$$
H_{2}(t, x) \equiv T_{s} x+C(t \lambda, x)+\varepsilon J x .
$$

Using the fact that $\left(T_{s}+\varepsilon J\right)(0)=0$, we note that $0 \notin H_{2}(t, \partial G)$ for any $t \in$ $[0,1]$. Following Browder [4, $H_{2}(t, x)$ is a homotopy of class $\left(S_{+}\right)$if the following condition holds: for any sequence $\left\{u_{j}\right\} \subset \bar{G}$ with $u_{j} \rightarrow u_{0}$ and any sequence $\left\{t_{j}\right\} \subset[0,1]$ with $t_{j} \rightarrow t_{0}$ for which we have

$$
\limsup _{j \rightarrow \infty}\left\langle H_{2}\left(t_{j}, u_{j}\right), u_{j}-u_{0}\right\rangle \leq 0,
$$

we also have $u_{j} \rightarrow u_{0}$ and $H_{2}\left(t_{j}, u_{j}\right) \rightarrow H_{2}\left(t_{0}, u_{0}\right)$. We are going to show that $H_{2}(t, x)$ is actually a homotopy of class $\left(S_{+}\right)$. To this end, we let $\left\{t_{j}\right\},\left\{u_{j}\right\}$ be as above. Then

(14) $\limsup _{j \rightarrow \infty}\left\langle H_{2}\left(t_{j}, u_{j}\right), u_{j}-u_{0}\right\rangle=\limsup _{j \rightarrow \infty}\left\langle T_{s} u_{j}+C\left(t_{j} \lambda, u_{j}\right)+\varepsilon J u_{j}, u_{j}-u_{0}\right\rangle \leq 0$.

We observe that

$$
\begin{aligned}
\left\langle H_{2}\left(t_{j}, u_{j}\right), u_{j}-\right. & \left.u_{0}\right\rangle \\
= & \left\langle T_{s} u_{j}, u_{j}-u_{0}\right\rangle+\left\langle C\left(t_{j} \lambda, u_{j}\right), u_{j}-u_{0}\right\rangle+\varepsilon\left\langle J u_{j}, u_{j}-u_{0}\right\rangle \\
= & \left\langle T_{s} u_{j}-T_{s} u_{0}, u_{j}-u_{0}\right\rangle+\left\langle T_{s} u_{0}, u_{j}-u_{0}\right\rangle \\
& +\left\langle C\left(t_{j} \lambda, u_{j}\right), u_{j}-u_{0}\right\rangle \\
& +\varepsilon\left\langle J u_{j}-J u_{0}, u_{j}-u_{0}\right\rangle+\varepsilon\left\langle J u_{0}, u_{j}-u_{0}\right\rangle \\
\geq & \left\langle T_{s} u_{0}, u_{j}-u_{0}\right\rangle+\left\langle C\left(t_{j} \lambda, u_{j}\right), u_{j}-u_{0}\right\rangle+\varepsilon\left\langle J u_{0}, u_{j}-u_{0}\right\rangle .
\end{aligned}
$$

Using this in (14) we obtain

$$
\limsup _{j \rightarrow \infty}\left\langle C\left(t_{j} \lambda, u_{j}\right), u_{j}-u_{0}\right\rangle \leq 0 .
$$


If $t_{0}=0$, then $C\left(t_{j} \lambda, u_{j}\right) \rightarrow 0$ and

$$
\lim _{j \rightarrow \infty}\left\langle C\left(t_{j} \lambda, u_{j}\right), u_{j}-u_{0}\right\rangle=0 .
$$

Using this and the monotonicity of $T_{s}$ in the first equality of (15) we obtain

$$
\limsup _{j \rightarrow \infty}\left\langle J u_{j}, u_{j}-u_{0}\right\rangle \leq 0 .
$$

Since $J$ is of type $\left(S_{+}\right)$, we have $u_{j} \rightarrow u_{0}$, which implies $T_{s} u_{j} \rightarrow T_{s} u_{0}, C\left(t_{j} \lambda, u_{j}\right) \rightarrow$ $C\left(0, u_{0}\right)=0$ and $J u_{j} \rightarrow J u_{0}$. This says that

$$
H_{2}\left(t_{j}, u_{j}\right) \rightarrow H_{2}\left(0, u_{0}\right)=T_{s} u_{0}+\varepsilon J u_{0}
$$

and the proof for the case $t_{0}=0$ is complete.

Now, let $t_{0}>0$. We have

$$
\left\langle C\left(t_{j} \lambda, u_{j}\right), u_{j}-u_{0}\right\rangle=\left\langle C\left(t_{j} \lambda, u_{j}\right)-C\left(t_{0} \lambda, u_{j}\right), u_{j}-u_{0}\right\rangle+\left\langle C\left(t_{0} \lambda, u_{j}\right), u_{j}-u_{0}\right\rangle,
$$

which implies

$$
\begin{aligned}
\limsup _{j \rightarrow \infty}\left\langle C\left(t_{0} \lambda, u_{j}\right), u_{j}-u_{0}\right\rangle \leq & \limsup _{j \rightarrow \infty}\left\langle C\left(t_{j} \lambda, u_{j}\right), u_{j}-u_{0}\right\rangle \\
& +\limsup _{j \rightarrow \infty}\left\{-\left\langle C\left(t_{j} \lambda, u_{j}\right)-C\left(t_{0} \lambda, u_{j}\right), u_{j}-u_{0}\right\rangle\right\} .
\end{aligned}
$$

By (16), this yields

$$
\limsup _{j \rightarrow \infty}\left\langle C\left(t_{0} \lambda, u_{j}\right), u_{j}-u_{0}\right\rangle \leq \lim _{j \rightarrow \infty}\left\|C\left(t_{j} \lambda, u_{j}\right)-C\left(t_{0} \lambda, u_{j}\right)\right\|\left\|u_{j}-u_{0}\right\|=0 .
$$

By the $\left(S_{+}\right)$-property of $C$, we get $u_{j} \rightarrow u_{0}, T_{s} u_{j} \rightarrow T_{s} u_{0}, C\left(t_{j} \lambda, u_{j}\right) \rightarrow C\left(t_{0} \lambda, u_{0}\right)$ and $J u_{j} \rightarrow J u_{0}$. Consequently, $H_{2}\left(t_{j}, u_{j}\right) \rightarrow H_{2}\left(t_{0}, u_{0}\right)$. This finishes the proof of the fact that $H_{2}$ is a homotopy of class $\left(S_{+}\right)$. Thus,

$$
\begin{aligned}
d_{S}\left(H_{2}(t, \cdot), G, 0\right) & =d_{S}\left(H_{2}(1, \cdot), G, 0\right) \\
& =d_{S}\left(H_{2}(0, \cdot), G, 0\right)=d_{S}\left(T_{s}+\varepsilon J, G, 0\right)=1,
\end{aligned}
$$

where $d_{S}$ denotes the Skrypnik degree (cf. [22]-23]). The last equality in (18) comes from [6, Theorem 3, (iv)]. In fact, the mapping $T_{s}+\varepsilon J$ is demicontinuous, injective and of type $\left(S_{+}\right)$on $\bar{G}$, and such that

$$
\left\langle T_{s} x+\varepsilon J x, x\right\rangle \geq 0, \quad x \in \partial G .
$$

For Browder's degree $d_{B}$ in [5] we have, in our setting,

$$
d_{B}(H(\lambda, \cdot), G, 0)=\lim _{s \downarrow 0} d_{S}\left(H_{1}(s, \lambda, \cdot), G, 0\right)=\lim _{s \downarrow 0} d_{S}\left(H_{2}(1, \cdot), G, 0\right)=1
$$

because $H_{1}(s, \lambda, x)=H_{2}(1, x)$. Thus, by Browder's degree theory,

$$
0 \in(T+C(\lambda, \cdot)+\varepsilon J)(D(T) \cap G),
$$

which contradicts our assumed property $(\mathcal{P})$. Therefore, $(2)$ is true.

(ii) Let the sequences $\left\{x_{n}\right\} \subset D(T) \cap \partial G, u_{n}^{*} \in T x_{n}, \lambda_{n} \in(0, \Lambda]$ be such that

$$
u_{n}^{*}+C\left(\lambda_{n}, x_{n}\right)+(1 / n) J x_{n}=0 .
$$

We may assume that $\lambda_{n} \rightarrow \lambda_{0} \in[0, \Lambda], x_{n} \rightarrow x_{0}, C\left(\lambda_{n}, x_{n}\right) \rightarrow c^{*}$ and $J x_{n} \rightarrow j^{*}$. We consider two cases:

(j) $\lambda_{0}=0$

(jj) $\lambda_{0}>0$. 
(j) Since for some $u_{n}^{*} \in T x_{n}$ we have $u_{n}^{*}=-C\left(\lambda_{n}, x_{n}\right)-(1 / n) J x_{n} \rightarrow 0$ and $T$ satisfies condition $\left(S_{q}\right)$, we have $x_{n} \rightarrow x_{0} \in \partial G$. The closedness of $T$ (see Lemma A) now implies $0 \in T x_{0}$, which contradicts $0 \notin T(D(T) \cap \partial G)$.

(jj) We are going to show first that

$$
\limsup _{n \rightarrow \infty}\left\langle C\left(\lambda_{n}, x_{n}\right), x_{n}-x_{0}\right\rangle \leq 0 .
$$

Assume the contrary. Then we may also choose $\left\{x_{n}\right\}$, or a subsequence of it denoted again by $\left\{x_{n}\right\}$, so that

$$
\lim _{n \rightarrow \infty}\left\langle C\left(\lambda_{n}, x_{n}\right), x_{n}-x_{0}\right\rangle>0
$$

We have

$$
\left\langle u_{n}^{*}, x_{n}-x_{0}\right\rangle=-\left\langle C\left(\lambda_{n}, x_{n}\right), x_{n}-x_{0}\right\rangle-\left\langle(1 / n) J x_{n}, x_{n}-x_{0}\right\rangle,
$$

which says

$$
\limsup _{n \rightarrow \infty}\left\langle u_{n}^{*}, x_{n}-x_{0}\right\rangle<0 .
$$

Since, by $(21), u_{n}^{*} \rightarrow-c^{*}$, we also have

$$
\left\langle u_{n}^{*}, x_{n}\right\rangle=\left\langle u_{n}^{*}, x_{n}-x_{0}\right\rangle+\left\langle u_{n}^{*}, x_{0}\right\rangle
$$

and

$$
\limsup _{n \rightarrow \infty}\left\langle u_{n}^{*}, x_{n}\right\rangle<\left\langle-c^{*}, x_{0}\right\rangle .
$$

Now, we fix $\left(x, x^{*}\right) \in G(T)$ and examine

$$
\left\langle u_{n}^{*}-x^{*}, x_{n}-x\right\rangle \geq 0 .
$$

We obtain

$$
\left\langle u_{n}^{*}, x_{n}\right\rangle \geq\left\langle u_{n}^{*}, x\right\rangle+\left\langle x^{*}, x_{n}-x\right\rangle
$$

which implies

$$
\liminf _{n \rightarrow \infty}\left\langle u_{n}^{*}, x_{n}\right\rangle \geq\left\langle-c^{*}, x\right\rangle+\left\langle x^{*}, x_{0}-x\right\rangle .
$$

Combining this and (25), we find that

$$
\left\langle-c^{*}-x^{*}, x_{0}-x\right\rangle>0 .
$$

Since $T$ is maximal monotone and $\left(x, x^{*}\right) \in G(T)$ is arbitrary, we get $x_{0} \in D(T)$ and $-c^{*} \in T x_{0}$. However, letting $x=x_{0}$ in (26) we get a contradiction. Thus, (22) is true. We observe that

$$
\left\langle C\left(\lambda_{n}, x_{n}\right), x_{n}-x_{0}\right\rangle=\left\langle C\left(\lambda_{n}, x_{n}\right)-C\left(\lambda_{0}, x_{n}\right), x_{n}-x_{0}\right\rangle+\left\langle C\left(\lambda_{0}, x_{n}\right), x_{n}-x_{0}\right\rangle .
$$

Using again the fact that $C\left(\lambda_{n}, x_{n}\right)-C\left(\lambda_{0}, x_{n}\right) \rightarrow 0$, we obtain

$$
\limsup _{n \rightarrow \infty}\left\langle C\left(\lambda_{0}, x_{n}\right), x_{n}-x_{0}\right\rangle \leq 0 .
$$

Since $C$ is of type $\left(S_{+}\right)$, we have $x_{n} \rightarrow x_{0} \in \partial G, C\left(\lambda_{n}, x_{n}\right) \rightarrow C\left(\lambda_{0}, x_{0}\right)=c^{*}$ and $u_{n}^{*} \rightarrow-C\left(\lambda_{0}, x_{0}\right)$. The demiclosedness of $T$ (see Lemma A) implies $T x_{0}+$ $C\left(\lambda_{0}, x_{0}\right) \ni 0$, and the proof of the theorem is complete. 
Theorem 1 has the following important corollary.

Corollary 1. Let $G \subset X$ be open and bounded. Let $T: X \supset D(T) \rightarrow 2^{X^{*}}$ be maximal monotone with $0 \in D(T) \cap G$ and $0 \in T(0)$. Let $C: \bar{G} \rightarrow X^{*}$ be demicontinuous, bounded and of type $\left(S_{+}\right)$. Let $\Lambda, \varepsilon$ and $\varepsilon_{0}$ be positive numbers. Assume that

$(\mathcal{P})$ there exists $\lambda \in(0, \Lambda]$ such that the inclusion

$$
T x+\lambda C x+\varepsilon J x \ni 0
$$

has no solution in $D(T) \cap G$. Then

(i) there exists $\left(\lambda_{0}, x_{0}\right) \in(0, \Lambda] \times(D(T) \cap \partial G)$ such that

$$
T x_{0}+\lambda_{0} C x_{0}+\varepsilon J x_{0} \ni 0 ;
$$

(ii) if $0 \notin T(D(T) \cap \partial G), T$ satisfies condition $\left(S_{q}\right)$ of $\partial G$, and property $(\mathcal{P})$ is satisfied for every $\varepsilon \in\left(0, \varepsilon_{0}\right]$, then there exists $\left(\lambda_{0}, x_{0}\right) \in(0, \Lambda] \times(D(T) \cap \partial G)$ such that $T x_{0}+\lambda_{0} C x_{0} \ni 0$.

Proof. It suffices to note that the operator $C(\lambda, x) \equiv \lambda C x$ has all the properties assumed for it in Theorem 1.

\section{Densely defined operators $T, C$}

In this section we apply the authors' degree theory from [13. for densely defined operators $T, C$.

Let $L$ be a subspace of $X$ and let $T: X \supset D(T) \rightarrow X^{*}$ be maximal monotone and $C: X \supset D(C) \rightarrow X^{*}$. Let $\mathcal{F}(L)$ be the set of all finite-dimensional subspaces of $L$. For the operator $T$ we consider the following assumptions:

$\left.t_{1}\right) T$ is monotone, i.e.

for every $u, v \in D(T)$. Moreover,

$$
\langle T u-T v, u-v\rangle \geq 0,
$$

$\left.t_{2}\right)$ for every $\left(u_{0}, h_{0}\right) \in X \times X^{*}$ with

$$
\left\langle T u-h_{0}, u-u_{0}\right\rangle \geq 0, \quad \text { for } u \in L,
$$

we have $u_{0} \in D(T)$ and $T u_{0}=h_{0}$;

$\left.t_{3}\right)$ for any $u_{0} \in D(T)$ we have

$$
\inf \left\{\left\langle T v-T u_{0}, v-u_{0}\right\rangle: v \in L\right\}=0 ;
$$

$t_{4}$ ) for every $F \in \mathcal{F}(L), v \in L$ the mapping $t(F, v): F \rightarrow \mathcal{R}$, defined by $t(F, v) u=\langle T u, v\rangle$ is continuous.

For the operator $C$ we have the following assumptions:

$\left.c_{1}\right)$

$$
L \subset D(C)
$$

and $C$ is quasi-bounded with respect to $T$, i.e. for every number $S>0$ there exists a number $K(S)>0$ such that from the inequalities

$$
\langle T u+C u, u\rangle \leq 0, \quad\|u\| \leq S, \quad u \in L,
$$

we have $\|C u\| \leq K(S)$; 
$\left.c_{2}\right)$ the operator $C$ satisfies the following generalized $\left(S_{+}\right)$condition with respect to $T$ : for every sequence $\left\{u_{n}\right\} \subset L$ such that $u_{n} \rightarrow u_{0}, C u_{n} \rightarrow h_{0}$ and

$$
\limsup _{n \rightarrow \infty}\left\langle C u_{n}, u_{n}-u_{0}\right\rangle \leq 0, \quad\left\langle T u_{n}+C u_{n}, u_{n}\right\rangle \leq 0
$$

for some $u_{0} \in X, h_{0} \in X^{*}$, we have $u_{n} \rightarrow u_{0}, u_{0} \in D(C)$ and $C u_{0}=h_{0}$;

$c_{3}$ ) for every $F \in \mathcal{F}(L), v \in L$ the mapping $c(F, v): F \rightarrow \mathcal{R}$, defined by $c(F, v)(u)=\langle C u, v\rangle$, is continuous.

Note that the conditions $\left.\left.t_{2}\right), t_{3}\right)$ are satisfied for a maximal monotone operator $T$ whose domain $D(T)=L$.

Remark 1. We should note here that the degree theory developed in 13 used the number $S$ in place of 0 in the first inequality of (32). A careful study on the development in 13 reveals that all we need is our present assumption. The same remark applies to the homotopy assumption $a_{t}^{(1)}$ in [13, p. 432]: we can replace $S$ in the first inequality there by 0 .

An operator $C: X \supset D(C) \rightarrow X^{*}$ with $L \subset D(C)$ is called " $L$-quasibounded" if for every $S>0$ there exists $K(S)>0$ such that $u \in L$ with $\|u\| \leq S,\langle C u, u\rangle \leq 0$ implies $\|C u\| \leq K(S)$.

Theorem 2. Let $G \subset X$ be open and bounded with $0 \in G$. Assume that the operator $T$ is single-valued and maximal monotone, $D(T)=L, T(0)=0$ and $T$ satisfies $\left.t_{4}\right)$, while the operator $C: X \supset D(C) \rightarrow X^{*}$ is L-quasibounded and satisfies $\left(\widetilde{S}_{+}\right)_{L}$ and $\left.c_{3}\right)$. Let $\varepsilon, \varepsilon_{0}$ and $\Lambda$ be positive numbers. Assume that

$(\mathcal{P})$ there exists $\lambda \in(0, \Lambda]$ such that the equation

$$
T x+\lambda C x+\varepsilon J x=0
$$

has no solution in $L \cap G$. Then

(i) there exists $\left(\lambda_{0}, x_{0}\right) \in(0, \Lambda] \times(L \cap \partial G)$ such that

$$
T x_{0}+\lambda_{0} C x_{0}+\varepsilon J x_{0}=0 ;
$$

(ii) if $0 \notin T(L \cap \partial G), T$ satisfies $\left(S_{q}\right)$ on $\partial G$, and property $(\mathcal{P})$ is satisfied for every $\varepsilon \in\left(0, \varepsilon_{0}\right]$, then there exists $\left(\lambda_{0}, x_{0}\right) \in(0, \Lambda] \times(L \cap \partial G)$ such that $T x_{0}+$ $\lambda_{0} C x_{0}=0$.

Before we prove this theorem, we notice that both conditions $\left.c_{1}\right), c_{2}$ ) are satisfied, because $\langle T u, u\rangle \geq 0$ for all $u \in D(T)$.

Proof. Assume that (35) is not true. Then for every $\lambda \in(0, \Lambda]$ the equation

$$
T x+\lambda C x+\varepsilon J x=0
$$

has no solution $x \in L \cap \partial G$. We consider the operators: $T_{t} \equiv T+(\varepsilon / 2) J$ and $C_{t} \equiv t \Lambda C+(\varepsilon / 2) J, \quad t \in[0,1]$. We need to show that $T_{t}+C_{t}$ is an admissible homotopy in the sense of Definition 4.3 in [13]. To this end, we show first the uniform quasiboundedness property of $C_{t}$ w.r.t. $T_{t}$. This is the property $a_{t}^{(1)}$ in [13] with the first occurrence of $S$ in (4.11) there replaced by 0 (see Remark 1 above). Assume that for some $S>0$ we have

$$
\left\langle T_{t} u+C_{t} u, u\right\rangle \leq 0, \quad\|u\| \leq S, \quad \text { for some } u \in L, t \in[0,1] .
$$


If $t=0$, then

$$
\left\|C_{0} u\right\|=(\varepsilon / 2)\|J u\| \leq(\varepsilon / 2) S
$$

If $t>0$, then

$$
\Lambda\langle C u, u\rangle \leq\langle(1 / t) T u+\Lambda C u, u\rangle \leq-\langle(1 / t) \varepsilon J u, u\rangle \leq 0
$$

and the $L$-quasiboundedness of $C$ gives $\|C u\| \leq K(S)$. This implies

$$
\left\|C_{t} u\right\| \leq K(S)+(\varepsilon / 2) S .
$$

Combining this with (37) we obtain an obvious uniform quasiboundedness constant $\widetilde{K}(S)$ for the operator $C_{t}$.

We now show the uniform generalized condition $\left(S_{+}\right)$of $C_{t}$ w.r.t. $T_{t}$, which is condition $a_{t}^{(2)}$ in [13. To this end, assume that $\left\{t_{n}\right\} \subset[0,1],\left\{u_{n}\right\} \subset L$ are such that $u_{n} \rightarrow u_{0}, C_{t_{n}} u_{n} \rightarrow h_{0}^{*}, t_{n} \rightarrow t_{0}$ and

$$
\limsup _{n \rightarrow \infty}\left\langle C_{t_{n}} u_{n}, u_{n}-u_{0}\right\rangle \leq 0,\left\langle T_{t_{n}}+C_{t_{n}} u_{n}, u_{n}\right\rangle \leq 0 .
$$

We rewrite (40) as follows:

(41) $\limsup _{n \rightarrow \infty}\left\langle t_{n} \Lambda C u_{n}+(\varepsilon / 2) J u_{n}, u_{n}-u_{0}\right\rangle \leq 0,\left\langle T u_{n}+t_{n} \Lambda C u_{n}+\varepsilon J u_{n}, u_{n}\right\rangle \leq 0$.

If $t_{n}=0$ for all large $n$, then the first of (41) implies

$$
\limsup _{n \rightarrow \infty}\left\langle J u_{n}, u_{n}-u_{0}\right\rangle \leq 0 .
$$

Since $J$ is of type $\left(S_{+}\right)$, this says that $u_{n} \rightarrow u_{0}$ and $J u_{n} \rightarrow J u_{0}$. Thus, $u_{0} \in$ $D\left(C_{0}\right)=X$ and $C_{0} u_{0}=(\varepsilon / 2) J u_{0}=h_{0}^{*}$.

Let $t_{n}=0$ for infinitely many $n$, but not all large $n$. Then $t_{n} \rightarrow 0$. Denote by $\left\{t_{n}\right\}$ again the subsequence of $\left\{t_{n}\right\}$ of positive terms. Then $t_{n} \rightarrow 0$ and, from the second part of (41) (since $\left.t_{n} \leq 1\right)$,

$$
\Lambda\left\langle C u_{n}, u_{n}\right\rangle \leq\left\langle\left(1 / t_{n}\right) T u_{n}+\Lambda C u_{n}+\left(\varepsilon / t_{n}\right) J u_{n}, u_{n}\right\rangle \leq 0 .
$$

Since $C$ is $L$-quasibounded, there exists a constant $K>0$ such that $\left\|C u_{n}\right\| \leq K$ for all $n$. It follows that, for the original sequence $\left\{t_{n}\right\}$, we have

$$
\lim _{n \rightarrow \infty} t_{n} \Lambda C u_{n}=0
$$

and

(45) $w-\lim _{n \rightarrow \infty} C_{t_{n}} u_{n}=w-\lim _{n \rightarrow \infty}\left[t_{n} \Lambda C u_{n}+(\varepsilon / 2) J u_{n}\right]=w-\lim _{n \rightarrow \infty}(\varepsilon / 2) J u_{n}=h_{0}^{*}$,

where " $w$ " denotes weak limit. Also, the first part of (41) implies (42), which implies again $u_{n} \rightarrow u_{0}$ and $J u_{n} \rightarrow J u_{0}$. Once again, we have

$$
u_{0} \in D\left(C_{0}\right)=X \text { and } C_{0} u_{0}=(\varepsilon / 2) J u_{0}=h_{0}^{*} .
$$

It remains to consider the case $t_{n}>0$ for all large $n$. We assume that $t_{n}>0$ for all $n$. If $t_{n} \rightarrow 0$, we repeat the above argument to obtain (46). Let us assume $t_{0}>0$. Then

$$
\begin{aligned}
\left\langle t_{n} \Lambda C u_{n}, u_{n}-u_{0}\right\rangle= & \left\langle t_{n} \Lambda C u_{n}+(\varepsilon / 2) J u_{n}, u_{n}-u_{0}\right\rangle-(\varepsilon / 2)\left\langle J u_{n}, u_{n}-u_{0}\right\rangle \\
= & \left\langle t_{n} \Lambda C u_{n}+(\varepsilon / 2) J u_{n}, u_{n}-u_{0}\right\rangle-(\varepsilon / 2)\left\langle J u_{n}-J u_{0}, u_{n}-u_{0}\right\rangle \\
& -(\varepsilon / 2)\left\langle J u_{0}, u_{n}-u_{0}\right\rangle, \\
\leq & \left\langle t_{n} \Lambda C u_{n}+(\varepsilon / 2) J u_{n}, u_{n}-u_{0}\right\rangle-(\varepsilon / 2)\left\langle J u_{0}, u_{n}-u_{0}\right\rangle
\end{aligned}
$$


which, in view of (41), implies

$$
\begin{aligned}
\limsup _{n \rightarrow \infty}\left\langle t_{n} \Lambda C u_{n}, u_{n}-u_{0}\right\rangle \leq & \limsup _{n \rightarrow \infty}\left\langle t_{n} \Lambda C u_{n}+(\varepsilon / 2) J u_{n}, u_{n}-u_{0}\right\rangle \\
& +\limsup _{n \rightarrow \infty}\left\{-(\varepsilon / 2)\left\langle J u_{0}, u_{n}-u_{0}\right\rangle\right\} \\
= & \limsup _{n \rightarrow \infty}\left\langle t_{n} \Lambda C u_{n}+(\varepsilon / 2) J u_{n}, u_{n}-u_{0}\right\rangle \\
& +\lim _{n \rightarrow \infty}\left\{-(\varepsilon / 2)\left\langle J u_{0}, u_{n}-u_{0}\right\rangle\right\} \leq 0 .
\end{aligned}
$$

If we assume that

$$
q \equiv \limsup _{n \rightarrow \infty}\left\langle C u_{n}, u_{n}-u_{0}\right\rangle>0,
$$

then there exists a subsequence $\left\{u_{n_{k}}\right\}$ of $\left\{u_{n}\right\}$ such that

$$
\lim _{k \rightarrow \infty}\left\langle C u_{n_{k}}, u_{n_{k}}-u_{0}\right\rangle=q \text {. }
$$

Then

$$
0<t_{0} \Lambda q=\lim _{n \rightarrow \infty} t_{n_{k}}\left\langle\Lambda C u_{n_{k}}, u_{n_{k}}-u_{0}\right\rangle \leq \limsup _{n \rightarrow \infty}\left\langle t_{n} \Lambda C u_{n}, u_{n}-u_{0}\right\rangle \leq 0,
$$

i.e. a contradiction. Consequently,

$$
\limsup _{n \rightarrow \infty}\left\langle C u_{n}, u_{n}-u_{0}\right\rangle \leq 0
$$

Since (43) and the $L$-quasiboundedness property of $C$ imply that $\left\{\left\|C u_{n}\right\|\right\}$ is bounded, we may assume that $C u_{n} \rightarrow h_{1}^{*}$. Since from the second part of (41) we obtain (43), we use the $\left(\widetilde{S}_{+}\right)_{L}$-property of $C$ to obtain that $u_{n} \rightarrow u_{0} \in D(C)$ and $C u_{0}=h_{1}^{*}$. Thus, $t_{0} \Lambda C u_{0}=h_{0}^{*}-(\varepsilon / 2) J u_{0}$. Thus, we have actually shown the following: every subsequence of $\left\{u_{n}\right\}$ contains a further subsequence, denoted again by $\left\{u_{n}\right\}$, such that $u_{n} \rightarrow u_{0}, u_{0} \in D\left(C_{t_{0}}\right)$ and $C_{t_{0}} u_{0}=h_{0}^{*}$. This implies that the original sequence $\left\{u_{n}\right\}$ has this property. The rest of the required conditions for the admissibility of our homotopy are trivially true. If follows that $T_{t}+C_{t}$ is an admissible homotopy for our degree $d$ in [13. This implies

$$
d\left(T_{t}+C_{t}, G, 0\right)=d(T+t \Lambda C+\varepsilon J, G, 0)=d(T+\varepsilon J, G, 0)=1 .
$$

The last equality follows from the fact that

$$
d(t(T+\varepsilon J)+(1-t) \varepsilon J, G, 0)=d(T+\varepsilon J, G, 0)=d(\varepsilon J, G, 0)=1 .
$$

The first degree above is well defined because

$$
(t(T+\varepsilon J)+(1-t) \varepsilon J)(L \cap \partial G)=(t T+\varepsilon J)(L \cap \partial G) \not \supset 0
$$

due to the fact that the mapping $t T+J$ is strictly and maximal monotone with $0 \in(t T+J)(L \cap G)$. This degree is also constant. In fact, the homotopy $H(t, x)=$ $t(T+\varepsilon J)+(1-t) \varepsilon J$ has already been used in [10, Proof of Theorem 3.1]. The operator $B+(1 / n) J-s$ there can be easily replaced by the operator $B=(\varepsilon / 2) J$ plus the operator $(\varepsilon / 2) J$. The last equality in (52) follows from Browder's Theorem 3 , (iv) in [6] because $0 \in \varepsilon J(G)$, and the mapping $\varepsilon J$ is demicontinuous, bounded, of type $\left(S_{+}\right)$, and such that $\langle\varepsilon J x, x\rangle \geq 0, x \in \partial G$. From our degree theory in 13 we obtain that equation (34) has a solution $(\lambda, x)$ in $L \cap G$ for every $\lambda \in(0, \Lambda]$. This contradicts $(\mathcal{P})$ and proves (i).

(ii) We have

$$
T x_{n}+\lambda_{n} C x_{n}+(1 / n) J x_{n}=0,
$$


where $\left\{x_{n}\right\} \subset L \cap \partial G,\left\{\lambda_{n}\right\} \subset(0, \Lambda]$. We may assume that $\lambda_{n} \rightarrow \lambda_{0} \in[0, \Lambda], x_{n} \rightarrow$ $x_{0}$ and $J x_{n} \rightarrow j^{*}$. We consider two cases:

(s) $\lambda_{0}=0$

(ss) $\lambda_{0}>0$.

We first note that we cannot have $\lambda_{n}=0$ for any $n$. This is due to the fact that $0 \in G, 0=(T+(1 / n) J)(0)$ and the operator $T+(1 / n) J$ is strictly monotone. Thus, $\lambda_{n}>0$ for all $n$. Using this in (51) we obtain $\left\langle C x_{n}, x_{n}\right\rangle \leq 0$ and the boundedness of $\left\{\left\|C x_{n}\right\|\right\}$.

(s) From (54) we also obtain

$$
T x_{n}=-\lambda_{n} C x_{n}-(1 / n) J x_{n} \rightarrow 0 .
$$

Since $T$ is of type $\left(S_{q}\right)$, we have $x_{n} \rightarrow x_{0} \in \partial G$. Since $T$ is closed (see Lemma A), $x_{0} \in D(T)=L$ and $T x_{0}=0$. Since $x_{0} \in L \cap \partial G$, we have a contradiction to our assumption on $T$. Thus, case (s) is impossible.

(ss) We may assume that $C x_{n} \rightarrow c^{*} \in X^{*}$. We have $T x_{n} \rightarrow-\lambda_{0} c^{*}$. We can now establish (50) exactly as in the last part of the proof of Theorem 1 . The $\left(\widetilde{S}_{+}\right)_{L^{-}}$ property of $C$ implies $x_{n} \rightarrow x_{0} \in \partial G, x_{0} \in D(C)$ and $C x_{n} \rightarrow C x_{0}=c^{*}$. Since $T x_{n} \rightarrow-\lambda_{0} C x_{0}$, the demiclosedness of $T$ implies $T x_{0}+\lambda_{0} C x_{0}=0$ with $\lambda_{0}>0$ and $x \in L \cap \partial G$. This completes the proof.

Actually, if $\Lambda \leq 1$ in Theorem 2, then that theorem holds under the assumptions $\left.c_{1}\right)-c_{3}$ ) for the operator $C$. This is the content of Theorem 3 below.

Theorem 3. Let $G \subset X$ be open and bounded with $0 \in G$. Assume that the operator $T$ is single-valued and maximal monotone, $D(T)=L, T(0)=0$ and $T$ satisfies $\left.t_{4}\right)$, while the operator $C$ satisfies $\left.\left.c_{1}\right)-c_{3}\right)$. Let $\varepsilon, \varepsilon_{0}$ and $\Lambda$ be positive numbers with $\Lambda \in(0,1]$. Assume that

$(\mathcal{P})$ there exists $\lambda \in(0, \Lambda]$ such that the equation

$$
T x+\lambda C x+\varepsilon J x=0
$$

has no solution in $L \cap G$. Then

(i) there exists $\left(\lambda_{0}, x_{0}\right) \in(0, \Lambda] \times(L \cap \partial G)$ such that

$$
T x_{0}+\lambda_{0} C x_{0}+\varepsilon J x_{0}=0 ;
$$

(ii) if $0 \notin T(L \cap \partial G)$, $T$ satisfies $\left(S_{q}\right)$ on $\partial G$, and property $(\mathcal{P})$ is satisfied for every $\varepsilon \in\left(0, \varepsilon_{0}\right]$, then there exists $\left(\lambda_{0}, x_{0}\right) \in(0, \Lambda] \times(L \cap \partial G)$ such that $T x_{0}+$ $\lambda_{0} C x_{0}=0$.

Proof. We just mention here that when $\Lambda \in(0,1]$ we may replace $C$ by $T+C$ in the relevant inequalities in the proof of Theorem 2. For example, the inequality (39) will now be replaced by

$$
\langle T u+C u, u\rangle \leq\langle[1 /(t \Lambda)] T u+C u, u\rangle \leq-\langle[1 /(t \Lambda)] \varepsilon J u, u\rangle \leq 0,
$$

while (43) will be changed to

$$
\left\langle T u_{n}+C u_{n}, u_{n}\right\rangle \leq\left\langle[1 /(t \Lambda)] T u_{n}+C u_{n}, u_{n}\right\rangle \leq-\left\langle[1 /(t \Lambda)] \varepsilon J u_{n}, u_{n}\right\rangle \leq 0 .
$$




\section{Densely Defined Perturbations $C$}

Theorem 4 below uses a new degree that was introduced by the authors in [14]. In particular, this degree applies to certain generalized pseudomonotone perturbations of multivalued maximal monotone operators.

The following definitions are needed for the application of the new degree. We recall that $L$ is a fixed dense subspace of the space $X$.

An operator $C: X \supset D(C) \rightarrow X^{*}$ is called "quasibounded" if for every $S>0$ there exists $K(S)>0$ such that $u \in D(C)$ with $\|u\| \leq S,\langle C u, u\rangle \leq 0$ implies $\|C u\| \leq K(S)$.

An operator $C: X \supset D(C) \rightarrow X^{*}$ with $L \subset D(C)$ is said to be "generalized pseudomonotone" if $\left\{u_{n}\right\} \subset D(C), u_{n} \rightarrow u_{0}, C u_{n} \rightarrow h_{0}^{*}$ and

$$
\limsup _{n \rightarrow \infty}\left\langle C u_{n}, u_{n}-u_{0}\right\rangle \leq 0
$$

imply $u_{0} \in D(C), C u_{0}=h_{0}^{*}$ and $\left\langle C u_{n}, u_{n}\right\rangle \rightarrow\left\langle h_{0}^{*}, u_{0}\right\rangle$.

It is easy to see that if an operator $C$ satisfies $\left(\widetilde{S}_{+}\right)$, then it is generalized pseudomonotone.

We denote by $J_{\psi}$ the duality mapping with gauge function $\psi$. The function $\psi: \mathcal{R}_{+} \rightarrow \mathcal{R}_{+}$is continuous, strictly increasing and such that $\psi(0)=0$ and $\psi(r) \rightarrow \infty$ at $r \rightarrow \infty$. This mapping $J_{\psi}$ is continuous, bounded, surjective, strictly and maximal monotone, and satisfies condition $\left(S_{+}\right)$. Also,

$$
\left\langle J_{\psi} x, x\right\rangle=\psi(\|x\|)\|x\| \text { and }\left\|J_{\psi} x\right\|=\psi(\|x\|), x \in X .
$$

For these facts we refer to Petryshyn [18, pp. 32-33 and 132].

Theorem 4. Let $G \subset X$ be open and bounded with $0 \in G$. Assume that the operator $T: X \supset D(T) \rightarrow 2^{X^{*}}$ is maximal monotone with $0 \in D(T)$ and $0 \in T(0)$. Assume that the operator $C: X \supset D(C) \rightarrow X^{*}$ is quasibounded, with $L \subset D(C)$, and satisfies $\left(\widetilde{S}_{+}\right)$and $\left.c_{3}\right)$. Let $\varepsilon, \varepsilon_{0}$ and $\Lambda$ be positive numbers. Assume that

$(\mathcal{P})$ there exists $\lambda \in(0, \Lambda]$ such that the inclusion

$$
T x+\lambda C x+\varepsilon J_{\psi} x \ni 0
$$

has no solution in $D(T+C) \cap G$. Then

(i) there exists $\left(\lambda_{0}, x_{0}\right) \in(0, \Lambda] \times(D(T+C) \cap \partial G)$ such that

$$
T x_{0}+\lambda_{0} C x_{0}+\varepsilon J_{\psi} x_{0} \ni 0
$$

(ii) if $0 \notin T(D(T) \cap \partial G)$, $T$ satisfies $\left(S_{q}\right)$ on $\partial G$, and property $(\mathcal{P})$ is satisfied for every $\varepsilon \in\left(0, \varepsilon_{0}\right]$, then there exists $\left(\lambda_{0}, x_{0}\right) \in(0, \Lambda] \times(D(T+C) \cap \partial G)$ such that $T x_{0}+\lambda_{0} C x_{0} \ni 0$.

Before we prove this result, we should mention that conditions $\left.t_{1}\right)-t_{4}$ ) have now been replaced by the condition that $T$ is maximal monotone (possible multivalued), $L \subset D(T)$ and $0 \in T(0)$. Also, the conditions on $C$ are no longer involving the space $L$ or the operator $T$. The degree mapping to be applied here (see [15]) comes from

$$
d(T+C, G, 0)=\lim _{s \downarrow 0} d\left(T_{s}+C, G, 0\right),
$$

where the degree mapping on the right-hand side is our degree from [13, which is fixed for all small values of $s>0$. Finally, the domain of the operator $T$ is not necessarily just the subspace $L$. The reader will have no trouble in extending Theorems 2 and 3 to other situations suggested by Theorem 4 . 
Proof of Theorem 4. (i) Assume that $\mathcal{P}$ is true and that the conclusion is false. Then (61) has no solution $(\lambda, x) \in(0, \Lambda] \times(D(T+C) \cap \partial G)$. We consider the homotopy inclusion

$$
H(t, x) \equiv T x+t \Lambda C x+\varepsilon J_{\psi} x \ni 0, \quad t \in[0,1] .
$$

This inclusion has no solution $x \in D(H(t, \cdot)) \cap \partial G$ for $t \in(0,1]$. This is also true for $t=0$ because 0 is already in the set $\left(T+\varepsilon J_{\psi}\right)(0)$ and the operator $T+\varepsilon J_{\psi}$ is strictly monotone (and hence one-to-one). We are going to show that $H(t, x)$ is an admissible homotopy for this degree. We do this because this homotopy was not studied in [15].

We set $T^{t}=T$ and recall from the Introduction the properties of the operator $T_{t, s}=T_{s} \equiv\left(T^{-1}+s J^{-1}\right)^{-1}: X \rightarrow X^{*}, s>0$. The operator $T^{t}$ here should not be confused, for $t=0$, with the operator $T^{\{0\}}$ in the Introduction. We also set $J_{t, s}=J_{s} \equiv I-s J^{-1} T_{t, s}=I-s J^{-1} T_{s}: X \rightarrow X$ and $C^{t} \equiv t \Lambda C+J_{\psi}$. We have $D(H(0, \cdot))=D(T)$ and $D(H(t, \cdot))=D(T+C), t \in(0,1]$. We also set $D^{t}=D(t \Lambda C)=D(t C)$. We have $D^{0}=X$ and $D^{t}=D(C)$ for $t \in(0,1]$. Let $G$ be an open and bounded subset of $X$.

We know that the equation

$$
T^{t} x+C^{t} x \ni 0
$$

has no solution $x \in D(H(t, \cdot)) \cap \partial G$ for any $t \in[0,1]$. We consider the equation

$$
T_{s} x+t \Lambda C x+\varepsilon J_{\psi}=0,
$$

and show that there exists $s_{1}>0$ such that

$$
0 \notin\left(T_{s}+t \Lambda C+\varepsilon J_{\psi}\right)\left(D^{t} \cap \partial G\right), \quad(s, t) \in\left(0, s_{1}\right] \times[0,1] .
$$

Assume that this is not true, and let $\left\{s_{n}\right\} \subset(0, \infty),\left\{t_{n}\right\} \subset[0,1],\left\{x_{n}\right\} \subset \partial G$ be such that $s_{n} \downarrow 0, t_{n} \rightarrow t_{0}$ and $x_{n} \rightarrow x_{0}$, where $t_{0} \in[0,1]$ and $x_{0} \in X$, and

$$
T_{s_{n}} x_{n}+t_{n} \Lambda C x_{n}+\varepsilon J_{\psi} x_{n}=0 .
$$

Obviously, we cannot have $t_{n}=0$ for any $n$, because $\left(T_{s_{n}}+J_{\psi}\right)(0)=0$ and the operator $T_{s_{n}}+J_{\psi}$ is strictly monotone (hence one-to-one). Thus, $t_{n}>0$ for all $n$. From (68) we see that

$$
\left\langle C x_{n}, x_{n}\right\rangle=-\left(1 /\left[t_{n} \Lambda\right]\right)\left\langle T_{s_{n}} x_{n}+\varepsilon J_{\psi} x_{n}, x_{n}\right\rangle \leq 0 .
$$

This and the quasiboundedness of $C$ imply that $\left\{C x_{n}\right\}$ is bounded. We may thus assume that $C x_{n} \rightarrow h_{0}^{*}$.

If $t_{0}=0$, then from

$$
\varepsilon \psi\left(\left\|x_{n}\right\|\right)\left\|x_{n}\right\|=\left\langle\varepsilon J_{\psi} x_{n}, x_{n}\right\rangle \leq-t_{n} \Lambda\left\langle C x_{n}, x_{n}\right\rangle \rightarrow 0
$$

we obtain $x_{n} \rightarrow 0 \in \partial G$, which is a contradiction to $0 \in G$. It follows that $t_{0}>$ 0 . Since $\left\{T_{s_{n}} x_{n}\right\},\left\{J_{\psi} x_{n}\right\}$ are bounded, we may assume that $T_{s_{n}} x_{n} \rightarrow h_{1}^{*}$ and $J_{\psi} x_{n} \rightarrow h_{2}^{*}$ with $t_{0} \Lambda h_{0}^{*}=-h_{1}^{*}-\varepsilon h_{2}^{*}$.

We now claim that

$$
\limsup _{n \rightarrow \infty}\left\langle t_{n} \Lambda C x_{n}+\varepsilon J_{\psi} x_{n}, x_{n}-x_{0}\right\rangle \leq 0 .
$$

If this is not true, then there is a subsequence of $\left\{t_{n}\right\}$, denoted again by $\left\{t_{n}\right\}$, such that

$$
\lim _{n \rightarrow \infty}\left\langle t_{n} \Lambda C x_{n}+\varepsilon J_{\psi} x_{n}, x_{n}-x_{0}\right\rangle>0 .
$$


This implies

This and $T_{s_{n}} x_{n} \rightarrow h_{1}^{*}$ imply

$$
\lim _{n \rightarrow \infty}\left\langle T_{s_{n}} x_{n}, x_{n}-x_{0}\right\rangle<0 .
$$

$$
\lim _{n \rightarrow \infty}\left\langle T_{s_{n}} x_{n}, x_{n}\right\rangle<\left\langle h_{1}^{*}, x_{0}\right\rangle .
$$

We can now repeat the relevant part of the proof of Theorem 3, (ii) in [15] in order to obtain a contradiction. In fact, as in [15], we arrive at

$$
\left\langle h_{1}^{*}-y, x_{0}-x\right\rangle>0, \quad \text { for every } x \in D(T), y \in T x .
$$

Since $T$ is maximal monotone, we have $x_{0} \in D(T)$ and $h_{1}^{*}=-t_{0} \Lambda h_{0}^{*}-\varepsilon h_{2}^{*} \in$ $T x_{0}$. However, this is a contradiction because (71) does not hold for $x=x_{0}, y=$ $h_{1}^{*}$. Thus, (69) is true.

From (69) we easily obtain that there is a subsequence of $\{n\}$, denoted again by $\{n\}$, such that one of the following is true:

$$
\limsup _{n \rightarrow \infty}\left\langle t_{n} \Lambda C_{n} x_{n}, x_{n}-x_{0}\right\rangle \leq 0, \quad \limsup _{n \rightarrow \infty}\left\langle\varepsilon J_{\psi} x_{n}, x_{n}-x_{0}\right\rangle \leq 0 .
$$

Assume that the first one is true. Then, by the $\left(\widetilde{S}_{+}\right)$-property of $C, x_{n} \rightarrow x_{0}, x_{0} \in$ $D(C)$ and $C x_{0}=h_{0}^{*}$. Then since

$$
\lim _{n \rightarrow \infty}\left\langle t_{n} \Lambda C x_{n}+\varepsilon J_{\psi} x_{n}, x_{n}-x_{0}\right\rangle=0,
$$

we also get

$$
\lim _{n \rightarrow \infty}\left\langle T_{s_{n}} x_{n}, x_{n}-x_{0}\right\rangle=0
$$

which implies (70), but with an equality sign. Working again as in the argument following (70) (see [15, proof of Theorem 3, (ii)), we see that (71) holds now but for the " $\geq$ " sign. It follows that $x_{0} \in D(T)$ and $T x_{0} \ni-t_{0} \Lambda h_{0}^{*}-\varepsilon h_{2}^{*}=$ $-t_{0} \Lambda C x_{0}-\varepsilon J_{\psi} x_{0}$. This is a contradiction again because, by $x_{n} \rightarrow x_{0}$, we have $x_{0} \in \partial G$. We have shown the validity of (67). An analogous proof holds when the second part of $(72)$ is true.

We have shown that $H(t, x)$ is an admissible homotopy for our degree. We can now work as in Theorem 3 of [15] in order to show that $d(H(t, \cdot), G, 0)=$ const. In fact, our case here is easier because the operator $T_{t, s}$ in $[15$ is now independent of $t$. Thus,

$$
d(H(t, \cdot), G, 0)=d\left(T_{s}+\varepsilon J_{\psi}, G, 0\right)=1 .
$$

The last equality above follows from Theorem 3, (i) of [15]. Consequently, the inclusion $H(t, x) \ni 0$ has a solution in $G$ for each $t \in[0,1]$. In particular, this says that $T x+\lambda C x+\varepsilon J_{\psi} x \ni 0$ has a solution in $G$ for every $\lambda \in(0, \Lambda]$. This is a contradiction to $(\mathcal{P})$ and finishes the proof of (i).

(ii) Let $\lambda_{n} \in(0, \Lambda], x_{n} \in D(C) \cap \partial G$ be such that, for some $u_{n}^{*} \in T x_{n}$,

$$
u_{n}^{*}+\lambda_{n} C x_{n}+(1 / n) J_{\psi} x_{n} \ni 0 \text {. }
$$

Again, we cannot have $\lambda_{n}=0$ for any $n$. Since $\lambda_{n}>0$, we have $\left\langle C x_{n}, x_{n}\right\rangle \leq 0$, which implies the boundedness of $\left\{C x_{n}\right\}$. We may assume that $\lambda_{n} \rightarrow \lambda_{0}, x_{n} \rightarrow$ $x_{0}, C x_{n} \rightarrow h_{0}^{*}$. Then $u_{n}^{*} \rightarrow-\lambda_{0} h_{0}^{*}$. If $\lambda_{0}=0$, then (73) implies

$$
\lim _{n \rightarrow \infty} u_{n}^{*}=\lim _{n \rightarrow \infty}\left[-\lambda_{n} C x_{n}-(1 / n) J_{\psi} x_{n}\right]=0 .
$$

Since $T$ satisfies $\left(S_{q}\right)$, this says that $x_{n} \rightarrow x_{0} \in \partial G$. Now, we can invoke the demiclosedness of $T$ (see Lemma A) to obtain $x_{0} \in D(T)$ and $0 \in T x_{0}$. This however contradicts $0 \notin T(D(T) \cap \partial G)$. Consequently, $\lambda_{0}>0$. 
At this point we can repeat the method of proof of part (i) in order to get the inequality

$$
\limsup _{n \rightarrow \infty}\left\langle C x_{n}, x_{n}-x_{0}\right\rangle \leq 0 .
$$

Since $C$ satisfies $\left(\widetilde{S}_{+}\right)$, this implies $x_{n} \rightarrow x_{0}, x_{0} \in D(C)$ and $C x_{0}=h_{0}^{*}$. Using the demiclosedness of $T$ (see Lemma A), we obtain $x_{0} \in D(T) \cap \partial G$ and $T x_{0}+\lambda_{0} C x_{0} \ni$ 0 . The proof is complete.

\section{A Fredholm alternative}

The function $\widetilde{J}_{\gamma}$ below is the duality mapping of $X$ with gauge function $\phi(r) \equiv$ $r^{\gamma}$, where $\gamma>0$. We have

$$
\left\langle\widetilde{J}_{\gamma} x, x\right\rangle=\|x\|^{\gamma+1}, \quad\left\|\widetilde{J}_{\gamma} x\right\|=\|x\|^{\gamma} .
$$

Let us assume that $T: X \supset D(T) \rightarrow X^{*}, C: X \supset D(T) \rightarrow X^{*}$ are such that $0 \in D(T) \cap D(C)$ and $T(0)=C(0)=0$. Then a number $\lambda \in \mathcal{R}$ is called an "eigenvalue" of the pair $(T, C)$ if the equation $\lambda T x+C x=0$ has a nonzero solution in $D(T) \cap D(C)$. We denote by $\Lambda(T, C)$ the set of all eigenvalues of $(T, C)$.

Our purpose in this section is to give a Fredholm alternative result in the sense of Necas (cf. [8, p. 61]). The operators $T, C$ are now homogeneous of degree $\gamma \geq 1$. This result has an analogue for linear operators $C$ and $T=I$ mapping a Hilbert space $X$ into itself. In that setting our result implies that if $\lambda$ is not an eigenvalue of $C$ (i.e. $\lambda$ does not belong to the point spectrum of $C$ ), then the resolvent operator exists on all of $X$ and is bounded.

Theorem 5. Assume that $L$ is a dense subspace of $X$. Assume that $T: L \rightarrow X^{*}$ is maximal monotone and satisfies $\left.t_{4}\right)$. Assume that $C: X \supset D(C) \rightarrow X^{*}, L \subset D(C)$ and $C$ satisfies $\left.\left.c_{1}\right)-c_{3}\right)$, but $\left.c_{2}\right)$ is satisfied with 0 in the second part of (33) replaced by any, but fixed, number $S>0$. Assume that $T(0)=0, C(0)=0$, and that for every $x \in L$ and every $r>0$ we have $T(r x)=r^{\gamma} T x$ and $C(r x)=r^{\gamma} C x$, where $\gamma \geq 1$ is fixed. Assume that $\lambda \geq 1$ and that the equation

$$
\lambda T x+C x+\mu \widetilde{J}_{\gamma} x=0
$$

has only the zero solution for any $\mu>0$. Then if $\lambda \notin \Lambda(T, C)$, the operator $\lambda T+C$ is surjective.

Proof. We first show that there exists a constant $\nu>0$ and $\varepsilon_{0}>0$ such that

$$
\left\|\lambda T x+C x+\varepsilon \widetilde{J}_{\gamma} x\right\| \geq \nu\|x\|^{\gamma}, \quad \text { for all } x \in L, \varepsilon \in\left(0, \varepsilon_{0}\right] .
$$

If this is not true, there exist sequences $\left\{x_{n}\right\} \subset L$ and $\left\{\varepsilon_{n}\right\} \downarrow 0$ such that

$$
\begin{aligned}
\lim _{n \rightarrow \infty} \frac{1}{\left\|x_{n}\right\|^{\gamma}} \| \lambda T x_{n}+ & C x_{n}+\varepsilon_{n} \widetilde{J}_{\gamma} x_{n} \| \\
& =\lim _{n \rightarrow \infty}\left\|\lambda T\left(\frac{x_{n}}{\left\|x_{n}\right\|}\right)+C\left(\frac{x_{n}}{\left\|x_{n}\right\|}\right)+\varepsilon \widetilde{J}_{\gamma}\left(\frac{x_{n}}{\left\|x_{n}\right\|}\right)\right\|=0 .
\end{aligned}
$$

Letting $u_{n}=x_{n} /\left\|x_{n}\right\|$, we have $\left\|u_{n}\right\|=1$ and

$$
\lim _{n \rightarrow \infty}\left\|\lambda T u_{n}+C u_{n}+\varepsilon_{n} \widetilde{J}_{\gamma} u_{n}\right\|=0 .
$$

Since $u_{n}$ is bounded, we may assume that $u_{n} \rightarrow u_{0}$. We have

$$
v_{n}^{*} \equiv \lambda T u_{n}+C u_{n} \rightarrow 0 .
$$


Also, the boundedness of $\left\{u_{n}\right\}$, the inequality

$$
\left\langle T u_{n}+C u_{n}, u_{n}\right\rangle \leq\left\langle\lambda T u_{n}+C u_{n}, u_{n}\right\rangle=\left\langle v_{n}^{*}, u_{n}\right\rangle \leq\left\|v_{n}^{*}\right\|\left\|u_{n}\right\|=\left\|v_{n}^{*}\right\|
$$

and the quasiboundedness of $C$ w.r.t. $T$ imply the boundedness of the operator $C$. Thus, we may assume that $C u_{n} \rightarrow c^{*} \in X^{*}$. We now claim that

$$
\limsup _{n \rightarrow \infty}\left\langle C u_{n}, u_{n}-u_{0}\right\rangle \leq 0
$$

If this is not true, then there exists a subsequence of $\left\{u_{n}\right\}$, denoted by $\left\{u_{n}\right\}$ again such that

$$
\lim _{n \rightarrow \infty}\left\langle C u_{n}, u_{n}-u_{0}\right\rangle>0
$$

This and (76) imply

$$
\lambda \limsup _{n \rightarrow \infty}\left\langle T u_{n}, u_{n}-u_{0}\right\rangle=\limsup _{n \rightarrow \infty}\left[\left\langle v_{n}^{*}, u_{n}-u_{0}\right\rangle-\left\langle C u_{n}, u_{n}-u_{0}\right\rangle\right]<0 .
$$

Once again, at this point we invoke our argument starting at (22) in the proof of Theorem 1 in order to obtain a contradiction to (78) and the validity of (77). Now, in view of (77) and the inequality following (76), we invoke the generalized $\left(S_{+}\right)$property of $C$ w.r.t. $T$ in order to obtain $u_{n} \rightarrow u_{0} \in D(C), C u_{0}=c^{*}$. Since $\lambda T u_{n} \rightarrow-C u_{0}$, the demiclosedness of $\lambda T$ implies $\lambda T u_{0}+C u_{0}=0$. However, since $\left\|u_{0}\right\|=1$, we obtain a contradiction to our assumption $\lambda \notin \Lambda(T, C)$. It follows that (74) is true.

We now fix $p^{*} \in X^{*}$ and look at the homotopy equation

$$
H(t, x) \equiv t\left(\lambda T x+C x+\varepsilon \widetilde{J}_{\gamma} x-p^{*}\right)+(1-t) \varepsilon \widetilde{J}_{\gamma} x=0 .
$$

All the solutions of (79) are bounded for $t=1$. In fact, let $\left\{x_{n}\right\} \subset L,\left\|x_{n}\right\| \rightarrow \infty$ and

$$
\lambda T x_{n}+C x_{n}+\varepsilon \widetilde{J}_{\gamma} x_{n}-p^{*}=0 .
$$

We may assume that $\left\|x_{n}\right\|>0$. Dividing by $\left\|x_{n}\right\|^{\gamma}$ we obtain

$$
\lambda T u_{n}+C u_{n}+\varepsilon \widetilde{J}_{\gamma} u_{n}=p^{*} /\left\|x_{n}\right\| \equiv v_{n}^{*} \rightarrow 0 .
$$

Repeating the argument above about (76), we obtain again that $u_{n} \rightarrow u_{0} \in L$ and $\lambda T u_{0}+C u_{0}+\varepsilon \widetilde{J}_{\gamma} u_{0}=0$. Since $\left\|u_{0}\right\|=1$, this is a contradiction to (74). We also note that the only solution of (79) for $t=0$ is $x=0$. We show first that all solutions $x=x_{t}$ of equation (79) are bounded independently of $t \in[0,1]$. By what we have just showed, we may assume that $t \in(0,1)$.

Assume that our assertion is not true. Then there exist sequences $\left\{t_{n}\right\} \subset(0,1)$, $\left\{x_{n}\right\} \subset L$ such that $\left\|x_{n}\right\| \rightarrow \infty, t_{n} \rightarrow t_{0} \in[0,1]$ and

$$
t_{n}\left(\lambda T x_{n}+C x_{n}+\varepsilon \widetilde{J}_{\gamma} x_{n}-p^{*}\right)+\left(1-t_{n}\right) \varepsilon \widetilde{J}_{\gamma} x_{n}=0 .
$$

We distinguish two cases:

(j) $t_{0}=0$;

(jj) $t_{0}>0$.

(j) Since $t_{n}>0$, we have

$$
\lambda T x_{n}+C x_{n}-p^{*}+\frac{1}{t_{n}} \varepsilon \widetilde{J}_{\gamma} x_{n}=0 .
$$


Assuming, without loss of generality, that $\left\|x_{n}\right\| \geq 1$, we have

$$
\begin{aligned}
& \frac{1}{\left\|x_{n}\right\|^{\gamma}}\left(\lambda T x_{n}+C x_{n}-p^{*}\right)+\frac{1}{\left\|x_{n}\right\|^{\gamma}} \frac{1}{t_{n}} \varepsilon \widetilde{J}_{\gamma} x_{n} \\
& =\lambda T\left(\frac{x_{n}}{\left\|x_{n}\right\|}\right)+C\left(\frac{x_{n}}{\left\|x_{n}\right\|}\right)-\frac{p^{*}}{\left\|x_{n}\right\|^{\gamma}}+\frac{1}{t_{n}} \varepsilon \widetilde{J}_{\gamma}\left(\frac{x_{n}}{\left\|x_{n}\right\|}\right)=0 .
\end{aligned}
$$

Letting $u_{n} \equiv x_{n} /\left\|x_{n}\right\|$ and $q_{n} \equiv 1 / t_{n}$ in (82), we obtain $q_{n}>0, q_{n} \rightarrow+\infty$ and

$$
\lambda T u_{n}+C u_{n}-\left(p^{*} /\left\|x_{n}\right\|^{\gamma}\right)+q_{n} \widetilde{J}_{\gamma} u_{n}=0 .
$$

Since $T(0)=0$, we have $\left\langle T u_{n}, u_{n}\right\rangle \geq 0$. We also have $\left\langle\widetilde{J}_{\gamma} u_{n}, u_{n}\right\rangle=\left\|u_{n}\right\|^{\gamma+1}=1$. Thus,

$$
\begin{aligned}
\left\langle T u_{n}+C u_{n}, u_{n}\right\rangle & \leq\left\langle\lambda T u_{n}+C u_{n}, u_{n}\right\rangle \\
& =\left\langle p^{*} /\left\|x_{n}\right\|^{\gamma}, u_{n}\right\rangle-q_{n}\left\langle\widetilde{J}_{\gamma} u_{n}, u_{n}\right\rangle \leq\left\|p^{*}\right\|\left\|u_{n}\right\|=\left\|p^{*}\right\| .
\end{aligned}
$$

Since $C$ is quasibounded, $\left\|u_{n}\right\|=1$ and (84) imply that $\left\{C u_{n}\right\}$ and $\left\langle C u_{n}, u_{n}\right\rangle$ are bounded. Using this in (84) we obtain

$$
0 \leq\left\langle T u_{n}, u_{n}\right\rangle \leq-\left\langle C u_{n}, u_{n}\right\rangle+\left\|p^{*}\right\|-q_{n} \rightarrow-\infty .
$$

This contradiction covers the case $(\mathrm{j})$.

(jj) We are again working with (83) with $u_{n} \rightarrow u_{0}, C u_{n} \rightarrow c^{*}$. We now have $q_{n} \rightarrow q_{0} \equiv 1 / t_{0} \geq 1$. Once again, we claim that

$$
\limsup _{n \rightarrow \infty}\left\langle C u_{n}, u_{n}-u_{0}\right\rangle \leq 0
$$

If this is not true, then (83) implies

$$
\limsup _{n \rightarrow \infty}\left\langle T u_{n}, u_{n}-u_{0}\right\rangle \leq 0 .
$$

Following the argument about (22) in the proof of Theorem 1, we get $u_{0} \in L$, which, along with (83), implies (85), i.e. a contradiction. Since $C$ satisfies the generalized condition $\left(S_{+}\right)$w.r.t. $T$, we have $u_{n} \rightarrow u_{0}$ and $C u_{n} \rightarrow C u_{0}$. We also have $\widetilde{J}_{\gamma} u_{n} \rightarrow \widetilde{J}_{\gamma} u_{0}$ and $\lambda T u_{n} \rightarrow-C u_{0}-q_{0} \varepsilon \widetilde{J}_{\gamma} u_{0}$. By the demiclosedness of $\lambda T$, we get $\lambda T u_{0}+C u_{0}+q_{0} \varepsilon \widetilde{J}_{\gamma} u_{0}=0$. Since $u_{0} \in \partial B_{1}(0)$, we have a contradiction to our assumption about (E). It follows that all possible solutions of the homotopy equation (79) are bounded independently of $t \in[0,1]$. Assume that they all lie inside the ball $B_{K}(0)$, for some $K>0$. We remark that $H(t, x)$ is an admissible homotopy for our degree in [13. Because of this,

$$
\begin{aligned}
d\left(H(t, \cdot), B_{K}(0), 0\right) & =d\left(H(1, \cdot), B_{K}(0), 0\right) \\
& =d\left(H(0, \cdot), B_{K}(0), 0\right)=d\left(\widetilde{J}_{\gamma}, B_{K}(0), 0\right)=1 .
\end{aligned}
$$

The last equality above follows from the fact that $\widetilde{J}_{\gamma}$ is demicontinuous, bounded, satisfies $\left(S_{+}\right)$, is one-to-one on $\overline{B_{K}(0)}$ and such that $\left\langle\widetilde{J}_{\gamma} x, x\right\rangle \geq 0$ for every $x \in$ $\partial B_{K}(0)$. Here, we quote Browder [6, Theorem 3, (iv)]. It follows that the equation

$$
\lambda T x+C x+(1 / n) \widetilde{J}_{\gamma} x=p^{*}
$$

is solvable for all large $n$. We may assume that this is true for all $n \geq 1$. Let $\left\{x_{n}\right\} \subset L$ solve (86). Then

$$
\lambda T x_{n}+C x_{n}+(1 / n) \widetilde{J}_{\gamma} x_{n}=p^{*} .
$$


If we assume that $\left\{x_{n}\right\}$, or a subsequence of it denoted again by $\left\{x_{n}\right\}$, is such that $\left\|x_{n}\right\| \rightarrow \infty$, we can divide (87) by $\left\|x_{n}\right\|$ and arrive at (76) with

$$
v_{n}^{*} \equiv \lambda T u_{n}+C u_{n}=-(1 / n) \widetilde{J}_{\gamma} u_{n}+p^{*} /\left\|x_{n}\right\| \rightarrow 0,
$$

where $u_{n}=x_{n} /\left\|x_{n}\right\|$. Assuming that $u_{n} \rightarrow u_{0}$, we use (88) to arrive again at $u_{n} \rightarrow u_{0}$ with $\lambda T u_{0}+C u_{0}=0$, which is a contradiction to $\lambda \notin \Lambda(T, C)$.

It follows that $\left\{x_{n}\right\}$ in (87) is bounded. Since

$$
\left\langle T x_{n}+C x_{n}, x_{n}\right\rangle \leq\left\langle\lambda T x_{n}+C x_{n}, x_{n}\right\rangle \leq-(1 / n)\left\|x_{n}\right\|^{\gamma+1}+\left\|p^{*}\right\|\left\|x_{n}\right\|,
$$

the quasiboundedness of $C$ w.r.t. $T$ implies that $\left\{C x_{n}\right\}$ is bounded. We may assume that $x_{n} \rightarrow x_{0}$ and $C x_{n} \rightarrow c^{*}$. Again repeating the argument about (22) as in the proof of Theorem 1, we obtain (85). With (85), we use the generalized $\left(S_{+}\right)$-property of $C$ w.r.t. $T$ in order to obtain $x_{n} \rightarrow x_{0}, x_{0} \in D(C)$ and $C x_{n} \rightarrow C x_{0}$. Again using the demiclosedness of $\lambda T$, we obtain $x_{0} \in L$ and $\lambda T x_{0}+C x_{0}=p^{*}$, and the proof is finished.

It should be noted that the assumption that (E) has only the zero solution for any $\mu>0$ cannot be omitted, in its entirety, in Theorem 5. In fact, let $T x=a x^{m}, C x=$ $b|x|^{m}, \lambda=2, \gamma=m, b>2 a>0$. Here, $m$ is an odd positive integer. The equation

$$
\lambda T x+C x+\mu \widetilde{J}_{m} x=2 a x^{m}+b|x|^{m}+\mu x^{m}=(2 a+\mu) x^{m}+b|x|^{m}=0
$$

has every negative number $x$ as a solution for $\mu=b-2 a$ or $2 a+\mu=b$. Also, the operator $\lambda T x+C x=b|x|^{m}+2 a x^{m} \geq 2 a\left(|x|^{m}+x^{m}\right) \geq 0$ is not surjective. We note that $\lambda \notin \Lambda(T, C)$ because $\lambda T x+C x=0$ implies $x=0$. Here, we have used $\widetilde{J}_{m} x=\operatorname{grad}|x|^{m+1} /(m+1)=|x|^{m} \operatorname{grad}|x|=|x|^{m} \operatorname{sgn} x=x^{m}$, for all $x \neq 0$.

\section{Operators $C$ Defined on $\overline{D(T)}$}

In this section we are not assuming everywhere that, for the Banach space $X$, $X, X^{*}$ are locally uniformly convex. The following result was given by Guan and Kartsatos in [9].

Theorem A. Assume that $T: X \supset \bar{D} \rightarrow X$ is accretive, bounded and $C: \bar{D} \rightarrow X$ is compact, where $D$ is an open, bounded subset of $X$ with $0 \in D$. Assume that there exists a constant $c>0$ such that the equation $T x-C x+c x=0$ has no solution $x \in \bar{D}$ and let one of the following conditions be satisfied:

(i) $X^{*}$ is uniformly convex and $T$ is demicontinuous.

(ii) $T$ is continuous.

Then there exists $\left(\lambda_{0}, x_{0}\right) \in(0,1) \times \partial D$ such that $T x_{0}-\lambda_{0} C x_{0}+c x_{0}=0$. If, moreover, $0 \notin T(\partial D), T$ is $\phi$-expansive on $\partial D$ and $T x-C x+c x=0$ has no solution $x \in \bar{D}$ for all small $c>0$, there exists $\left(\lambda_{0}, x_{0}\right) \in(0,1) \times \partial D$ such that $T x_{0}-\lambda_{0} C x_{0}=0$.

$\mathrm{Li}$ and Huang gave two eigenvalue results in 16 extending Theorem A, where $T$ is maximal monotone and $C$ is compact or completely continuous. As was mentioned by Guan and Kartsatos in [9], as well as other authors before, a considerable amount of eigenvalue existence theory is based on a result which is a simple but fundamental consequence of the Leray-Schauder theory in combination with situations like theorem A above. According to this result, if the compact operator $C: \bar{G} \rightarrow X$ has no fixed points in $\bar{G}$, then there exists $\left(\lambda_{0}, x_{0}\right) \in(0,1) \times \partial G$ such that $\left(I-\lambda_{0} C\right) x_{0}=0$. It is easy to see that in this particular case we have 
$d(I-C, G, 0)=0$. Such considerations, with substantial extensions and refinements, were used by the authors in [12.

This section provides an eigenvalue result along these lines for operators $\frac{T, C \text {, }}{D(T)}$ where $T$ is maximal monotone with compact resolvents and $C$ is defined on $\overline{D(T)}$ and is continuous and bounded there. This result complements the two results of $\mathrm{Li}$ and Huang in [16.

The resolvents $(T+\varepsilon J)^{-1}$ of the maximal monotone operator $T$ are strongly continuous mappings for all $\varepsilon>0$. Also, if one of them is compact, then they all are (cf., e.g., Kartsatos [11]).

Theorem 6. Let $T: X \supset D(T) \rightarrow 2^{X^{*}}$ be maximal monotone with compact resolvents. Assume that $C: X \supset \overline{D(T)} \rightarrow X^{*}$ is continuous and bounded. Let $G \subset X$ be open and bounded and such that $0 \in D(T) \cap G$ and $0 \in T(0)$. Let $\varepsilon, \varepsilon_{0}, \Lambda$ be given positive numbers. Assume that

$(\mathcal{P})$ there exists $\lambda \in(0, \Lambda]$ such that the inclusion

$$
T x+\lambda C x+\varepsilon J x \ni 0
$$

has no solution in $D(T) \cap G$. Then

(i) there exists $\left(\lambda_{0}, x_{0}\right) \in(0, \Lambda] \times(D(T) \cap \partial G)$ such that

$$
T x_{0}+\lambda_{0} C x_{0}+\varepsilon J x_{0} \ni 0
$$

(ii) if $0 \notin T(D(T) \cap \partial G)$ and property $(\mathcal{P})$ is satisfied for every $\varepsilon \in\left(0, \varepsilon_{0}\right]$, then there exists $\left(\lambda_{0}, x_{0}\right) \in(0, \Lambda] \times(D(T) \cap \partial G)$ such that $T x_{0}+\lambda_{0} C x_{0} \ni 0$.

Proof. (i) We now consider the homotopy equation

$$
H(\lambda, u) \equiv u+\lambda C(T+\varepsilon J)^{-1} u=0,
$$

for $\lambda \in(0, \Lambda], u \in D \equiv(T+\varepsilon J)(D(T) \cap \bar{G}) \subset X^{*}$. We notice that when $u \in D$ we have

$$
C(T+\varepsilon J)^{-1} u \in C(D(T) \cap \bar{G}),
$$

where the set on the right is bounded. Consequently, every solution $u \in D$ of (91) satisfies

$$
\|u\| \leq \Lambda K, \quad \text { where } K=\sup _{x \in D(T) \cap \bar{G}}\{\|C x\|\} .
$$

We fix $s>\Lambda K$ and let $Q \equiv T+\varepsilon J$. We note that $Q$ is injective and surjective with a continuous inverse $Q^{-1}: X^{*} \rightarrow X$. This implies that $Q(D(T) \cap G)$ is open and $Q(D(T) \cap \bar{G})$ is closed in $X^{*}$. In addition,

$$
Q(D(T) \cap \bar{G})=Q(D(T) \cap G) \cup Q(D(T) \cap \partial G)
$$

and

$$
\overline{Q(D(T) \cap G)}=Q(D(T) \cap G) \cup \partial Q(D(T) \cap G)
$$

imply

$$
Q(D(T) \cap \bar{G}) \supset \overline{Q(D(T) \cap G)}
$$

and

$$
Q(D(T) \cap \partial G) \supset \partial Q(D(T) \cap G) .
$$

It follows that in order to solve $(91)$ in $(T+\varepsilon J)(D(T) \cap \bar{G})$, via Leray-Schauder degree theory, it suffices to consider it only for $u \in \overline{U \cap B_{s}(0)}$, where $U \equiv$ $(T+\varepsilon J)(D(T) \cap G)$. 
We note that the set $U \cap B_{s}(0)$ is open and bounded. We also note that the set $C(T+\varepsilon J)^{-1}\left(\overline{U \cap B_{s}(0)}\right)$ is compact by the compactness of the resolvent $(T+\varepsilon J)^{-1}$ and the continuity of $C$. Thus, the Leray-Schauder degree $d(H(\lambda, \cdot), U, 0)$ will be well defined for all $\lambda \in[0, \Lambda]$ if

$$
0 \notin(I+\lambda F)\left(\partial\left(U \cap B_{s}(0)\right)\right),
$$

where $F \equiv C(T+\varepsilon J)^{-1}: \overline{U \cap B_{s}(0)} \rightarrow X^{*}$. Note that $0 \in U \cap B_{s}(0)$. Since $\partial\left(U \cap B_{s}(0)\right) \subset \partial U \cup \partial B_{s}(0)$, we know that (95) is true for $\lambda=0$. We assume that $\lambda>0$ and that (90) is not true. Then a) for every $\lambda \in(0, \Lambda]$ equation (89) has no solution $x \in D(T) \cap \partial G$.

Let us assume that b) there is $\lambda \in(0, \Lambda]$ such that $(I+\lambda F) u=0$ has a solution $u \in \partial\left(U \cap B_{s}(0)\right)$. We know that $\|u\| \leq \Lambda K<s$, so that $u \notin \partial B_{s}(0)$. Actually, $u \notin \partial U$ either. In fact, if $u \in \partial U$, then (94) implies $u \in Q(D(T) \cap \partial G)=$ $(T+\varepsilon J)(D(T) \cap \partial G)$. Letting $u=y^{*}+\varepsilon J x$, with $x \in D(T) \cap \partial G$ and $y^{*} \in T x$, we see from (91) that

$$
y^{*}+\varepsilon J x+\lambda C x=0
$$

or

$$
T x+\lambda C x+\varepsilon J x \ni 0 .
$$

Since $x \in D(T) \cap \partial G$, we have a contradiction to our assumption a).

It follows that

$$
d(H(\lambda, \cdot), U, 0)=d(H(0, \cdot), U, 0)=1 .
$$

Consequently, the equation $H(\lambda, u) \equiv(I+\lambda F) u=0$ is solvable with $u \in$ $((T+\varepsilon J)(D(T) \cap G)) \cap B_{s}(0)$ for every $\lambda \in(0, \Lambda]$. This implies that equation (89) is solvable in $D(T) \cap G$ for every $\lambda \in(0, \Lambda]$, i.e. a contradiction to $(\mathcal{P})$. Consequently, (90) holds and (i) is true.

(ii) We may assume that there exists a sequence $\left\{\left(\lambda_{n}, x_{n}\right)\right\} \subset(0, \Lambda] \times(D(T) \cap \partial G)$ such that

$$
T x_{n}+\lambda_{n} C x_{n}+(1 / n) J x_{n} \ni 0, \quad n=1,2, \ldots
$$

Then

$$
x_{n}=(T+J)^{-1}\left[-\lambda_{n} C x_{n}+(1-(1 / n)) J x_{n}\right] .
$$

Since $\left\{x_{n}\right\}$ and $\left\{\lambda_{n} C x_{n}\right\}$ are bounded, the compactness of $(T+J)^{-1}$ implies that $\left\{x_{n}\right\}$ lies in a compact set. Thus, we may assume that $x_{n} \rightarrow x_{0} \in \overline{D(T)} \cap \partial G$. We may also assume that $\lambda_{n} \rightarrow \lambda_{0} \in[0, \Lambda]$. If $\lambda_{0}=0$, then the closedness of $T$ implies $x_{0} \in D(T)$ and $0 \in T x_{0}$. This however is a contradiction to our assumption $0 \notin T(D(T) \cap \partial G)$. Consequently, $\lambda_{0}>0$ and, again by the closedness of $T$, $x_{0} \in D(T)$ and $T x_{0}+\lambda_{0} C x_{0} \ni 0$. The proof is finished.

If the operator $C$ in Theorem 6 is assumed to be defined just on the open and bounded set $\bar{G}$, then it can be extended to an operator $\widetilde{C}$ on all of $X$ by Dugundji's theorem. The operator $\widetilde{C}$ is continuous and its range lies in the convex hull of the range of $C$. It is thus bounded. The proof of Theorem 6 goes through in this case, with $C$ replaced by $\widetilde{C}$, without any further modifications. Other versions of Theorem 6 include the case where the resolvents of $T$ are completely continuous and $C$ is bounded and demicontinuous. 


\section{Continuous BRAnChes of Eigenvectors}

It is easy to see that condition $(\mathcal{P})$ does not allow $C(\lambda, 0)=0$ in Theorem 1 , or $C(0)=0$ in Theorems $2-4$, where $\lambda$ is as in condition $(\mathcal{P})$. As it was easily shown in [9, Lemma 4.2] for accretive operators $T$ and $J_{\psi}=J$, if $T: X \supset D(T) \rightarrow 2^{X^{*}}$, $C: X \supset D(C) \rightarrow X^{*}$ are such that $|(T+\lambda C) x| \geq \alpha>0$ for $x \in D(T+C) \cap G$, then there exists $\varepsilon_{0}>0$ such that the inclusion $T x+\lambda C x+\varepsilon J_{\psi} x \ni 0$ has no solution $x$ in $D(T+C) \cap G$ for any $\varepsilon \in\left(0, \varepsilon_{0}\right)$. Here, $\lambda$ is a fixed positive number, and for a set $\mathrm{A},|A|=\inf \{\|x\| ; x \in A\}$. In fact, if the constant $\alpha$ is as above,

$$
\begin{gathered}
M=\sup \{\psi(\|x\|): x \in G\}, \\
\varepsilon_{0} \in(0, \alpha / M), \varepsilon \in\left(0, \varepsilon_{0}\right), x \in D(T+C) \cap G \text { and } y^{*} \in T x, \text { then } \\
\left\|y^{*}+\lambda C x+\varepsilon J_{\psi} x\right\| \geq|T x+\lambda C x|-\varepsilon_{0} \psi(\|x\|) \geq \alpha-\varepsilon_{0} M>\alpha-\alpha=0 .
\end{gathered}
$$

Thus, the assumption $(\mathcal{P})$ in several theorems above may be replaced by an assumption like

$\left(\mathcal{P}_{1}\right)$ there exists $\lambda \in(0, \Lambda]$ and $\alpha>0$ such that

$$
|T x+\lambda C x| \geq \alpha, \quad x \in D(T+C) \cap G .
$$

Condition $\left(\mathcal{P}_{1}\right)$ implies that $C(0) \neq 0$. The conclusion in this case is obvious.

Analogous remarks are valid for the implicit case $C=C(\lambda, x)$.

We are now going to show that the results of this paper allow for the existence of continuous branches of eigenvectors. We need the following definition.

Definition 1. Let $T: X \supset D(T) \rightarrow 2^{X^{*}}, C: \mathcal{R} \times X \supset D(C) \rightarrow X^{*}$, be given and consider the problem

$$
T x+C(\lambda, x) \ni 0 .
$$

An "eigenvector" $x$ is a solution of (97) for some "eigenvalue" $\lambda$ with $x \in D(T)$ and $(\lambda, x) \in D(C)$. We say that the nonzero eigenvectors of the problem (97) form a "continuous branch of infinite length" if there exists $r_{0}>0$ such that, for every $r \geq r_{0}$, the sphere $\partial B_{r}(0)$ contains at least one nonzero eigenvector of (97).

We give below a result according to which the problem

$$
T x+\lambda C x \ni 0
$$

has nonzero eigenvectors forming a continuous branch of infinite length. This is done in the setting of Theorem 4. Analogous results hold for the other eigenvalue problems studied above.

Theorem 7. Assume that the operator $T: X \supset D(T) \rightarrow 2^{X^{*}}$ is maximal monotone with $0 \in D(T)$ and $0 \in T(0)$. Assume that the operator $C: X \supset D(C) \rightarrow X^{*}$ is quasibounded, with $L \subset D(C)$, and satisfies $\left(\widetilde{S}_{+}\right)$and $\left.c_{3}\right)$. Let $\Lambda$ be a positive number. Assume that $T x \ni 0$ implies $x=0, T$ satisfies $\left(S_{q}\right)$ and

$\left(\mathcal{P}_{1}\right)$ there exist $\alpha>0$ and $\lambda \in(0, \Lambda]$ such that

$$
|T x+\lambda C x| \geq \alpha, \quad x \in D(T+C) .
$$

Then the nonzero eigenvectors of the problem (98) form a continuous branch of infinite length with corresponding eigenvalues $\lambda \in(0, \Lambda]$. 
Proof. Let $r_{0}>0$ be given. Let $\varepsilon_{0}>0$ be so small that $\varepsilon_{0} r_{0}<\alpha$. Then

$$
|T x+\lambda C x+\varepsilon J x| \geq \alpha-\varepsilon\|x\| \geq \alpha-\varepsilon_{0} r_{0}>0, \quad x \in D(T+C),
$$

implies that the inclusion

$$
T x+\lambda C x+\varepsilon J x \ni 0
$$

has no solution $x \in D(T+C) \cap B_{r_{0}}(0)$ for any $\varepsilon \in\left(0, \varepsilon_{0}\right]$. Since $0 \notin T\left(\partial B_{r_{0}}(0)\right)$ and $T$ is of type $\left(S_{q}\right)$, Theorem 4 implies the existence of a solution $x_{\lambda_{0}}$ $\in D(T+C) \cap \partial B_{r_{0}}(0)$, for some $\lambda_{0} \in(0, \Lambda]$. The same argument can be repeated for any number $r>r_{0}$ instead of $r_{0}$ itself. The proof is complete.

In the following result we assume that the operator $T$ is defined and bounded on all of $X$. We do this in order to demonstrate the fact that the assumption $|T x+C x| \geq \alpha$ may be replaced in this case by the assumption $\|C x\| \geq \alpha$ on $D(C)$. An operator $T: X \supset D(T) \rightarrow 2^{X^{*}}$ is called "bounded" if for every bounded set $M \subset X$ the set $\bigcup\{T x: x \in D(T) \cap M\}$ is bounded.

Theorem 8. Assume that the operator $T: D(T)=X \rightarrow 2^{X^{*}}$ is maximal monotone and bounded with $0 \in T(0)$. Assume that the operator $C: X \supset D(C) \rightarrow X^{*}$ is quasibounded, with $L \subset D(C)$, and satisfies $\left(\widetilde{S}_{+}\right)$and $\left.c_{3}\right)$. Assume that $T x \ni 0$ implies $x=0, T$ satisfies $\left(S_{q}\right)$ and there exists $\alpha>0$ such that

$$
\|C x\| \geq \alpha, \quad x \in D(C) .
$$

Then the nonzero eigenvectors of the problem (98) form a continuous branch of infinite length.

Proof. We show that the problem (98) possesses eigenvectors on the set $\partial B_{r}(0)$ for every $r>0$. To this end, we fix $r>0, \varepsilon>0$ and show first that there exists $\widetilde{\lambda}>0$ such that

$$
d\left(T+\widetilde{\lambda} C+\varepsilon J, B_{r}(0), 0\right)=0 .
$$

If this is not true, then there exists a sequence $\left\{\lambda_{n}\right\} \subset(0, \infty)$ such that $\lambda_{n} \rightarrow \infty$ and one of the following holds:

(i) the degree $d\left(T+\lambda_{n} C+\varepsilon J, B_{r}(0), 0\right)$ is not well defined;

(ii) $d\left(T+\lambda_{n} C+\varepsilon J, B_{r}(0), 0\right) \neq 0$.

In case (i) there exist eigenvectors $x_{n} \in \partial B_{r}(0)$ such that

$$
T x_{n}+\lambda_{n} C x_{n}+\varepsilon J x_{n} \ni 0 .
$$

In case (ii) there exist eigenvectors $x_{n} \in B_{r}(0)$ such that (104) holds. Thus, in either case, there exists a sequence $\left\{x_{n}\right\} \subset \overline{B_{r}(0)}$ such that (104) holds. However, this leads to a contradiction because $\left\|\lambda_{n} C x_{n}+\varepsilon J\right\| \geq \alpha \lambda_{n}-\varepsilon r \rightarrow \infty$, while the sets $T x_{n}$ lie in a fixed bounded set. Thus, (103) is true for some $\tilde{\lambda}>0$.

We consider the homotopy

$$
H(t, x) \equiv T x+t \widetilde{\lambda} C x+\varepsilon J x, \quad t \in[0,1], x \in D(H(t, \cdot)) .
$$

Either there exist $t_{0} \in[0,1]$ and $x_{t_{0}} \in \partial B_{r}(0)$ such that

$$
T x_{t_{0}}+t_{0} \tilde{\lambda} C x_{t_{0}}+\varepsilon J x_{t_{0}} \ni 0,
$$

or

$$
d(H(t, \cdot)=d(H(1, \cdot)=0=d(H(0, \cdot)=1, \quad t \in[0,1],
$$


i.e. a contradiction. The last equality in (107) follows from Theorem 3, (i), in 15. It follows that (106) is true. Naturally, we must have $t_{0} \neq 0$ in (106) because otherwise $0 \in(T+\varepsilon J)\left(\partial B_{r}(0)\right)$. This cannot happen because we already have $0 \in(T+\varepsilon J)(0)$ and $T+\varepsilon J$ is one-to-one.

From (106) we obtain sequences $\lambda_{n} \in(0, \infty),\left\{x_{n}\right\} \subset \partial B_{r}(0)$ such that

$$
T x_{n}+\lambda_{n} C x_{n}+(1 / n) J x_{n} \ni 0 .
$$

We may assume that $x_{n} \rightarrow x_{0}$. Again, $\left\{\lambda_{n}\right\}$ cannot contain a subsequence $\left\{\lambda_{n_{k}}\right\}$ such that $\lambda_{n_{k}} \rightarrow \infty$ as $k \rightarrow \infty$ because the sequence $\left\{T x_{n_{k}}+\left(1 / n_{k}\right) J x_{n_{k}}\right\}$ lies in a bounded set and $\lambda_{n_{k}}\left\|C x_{n_{k}}\right\| \rightarrow \infty$ as $k \rightarrow \infty$.

From the quasiboundedness of $C$ and

$$
\left\langle C x_{n}, x_{n}\right\rangle=-\left(1 / \lambda_{n}\right)\left\langle y_{n}^{*}+(1 / n) J x_{n}, x_{n}\right\rangle \leq 0,
$$

we obtain that $\left\{C x_{n}\right\}$ is bounded. Thus, we may assume that $C x_{n} \rightarrow c^{*} \in X^{*}$. Since the sequence $\left\{\lambda_{n}\right\}$ is bounded, we may assume that $\lambda_{n} \rightarrow \lambda_{0}$. Again, $\lambda_{0} \neq 0$ otherwise $\lambda_{n} C x_{n}+(1 / n) J x_{n} \rightarrow 0$ and the $\left(S_{q}\right)$-property of $T$ would imply that $x_{n} \rightarrow x_{0} \in \partial G$. Since $T$ is demiclosed, Lemma A would imply $T x_{0} \ni 0$. This is a contradiction to our assumption that $T x \ni 0$ implies $x=0$. It follows that $\lambda_{0}>0$ and we may also assume that $\lambda_{n}>0$ for all $n$.

It is now easy to see that

$$
\limsup _{n \rightarrow \infty}\left\langle C x_{n}, x_{n}-x_{0}\right\rangle \leq 0 .
$$

Arguments like this were used in the proofs of Theorems 3 and 4 . From the $\left(\widetilde{S}_{+}\right)$property of $C$ we conclude that $x_{n} \rightarrow x_{0}, x_{0} \in D(C)$ and $C x_{0}=c^{*}$. Thus, since $y_{n}^{*} \rightarrow-\lambda_{0} C x_{0}, x_{n} \rightarrow x_{0}$ and $T$ is demiclosed (see Lemma A), we obtain $x_{0} \in$ $D(C) \cap \partial B_{r}(0)$ and $T x_{0}+\lambda_{0} C x_{0} \ni 0$.

Since $r>0$ is arbitrary, the nonzero eigenvectors of problem (98) form a continuous branch of infinite length.

The following result is a variant of Theorem 8 .

Theorem 9. Assume that the operator $T: D(T)=X \rightarrow 2^{X^{*}}$ is maximal monotone and bounded. Assume that the operator $C: X \supset D(C) \rightarrow X^{*}$ is bounded, with $L \subset D(C)$, and satisfies $\left(\widetilde{S}_{+}\right)$and $\left.c_{3}\right)$. Assume that $T x \ni 0$ implies $x=0, T$ satisfies $\left(S_{q}\right)$ and there exists $r_{0}>0$ such that

$$
\inf \left\{\|C x\|: x \in D(C) \backslash B_{r_{0}}(0)\right\} \equiv \alpha>0
$$

and, for each $r \geq r_{0}$,

$$
\overline{\left\{\frac{C x}{\|C x\|}: x \in D(C) \cap \partial B_{r}(0)\right\}} \neq \partial B_{1}(0) .
$$

Then the nonzero eigenvectors of the problem (98) form a continuous branch of infinite length.

Proof. Fix $r \geq r_{0}$. Then, by (110), there exists $y_{0}^{*} \in \partial B_{1}(0)$ such that

$$
y_{0}^{*} \notin \overline{\left\{\frac{C x}{\|C x\|}: x \in D(C) \cap \partial B_{r}(0)\right\}} .
$$

We fix $\varepsilon>0$ and show that there exists $\tilde{\lambda}>0$ such that

$$
(T+\widetilde{\lambda} C+\varepsilon J) x \not \supset \eta y_{0}^{*}, \quad(\eta, x) \in(0, \infty) \times\left(D(T+C) \cap \partial B_{r}(0)\right) .
$$


Assume that this is not true. Then there exist sequences $\left\{\lambda_{n}\right\} \subset(0, \infty), \eta_{n} \subset$ $(0, \infty),\left\{x_{n}\right\} \subset D(T+C) \cap \partial B_{r}(0), y_{n}^{*} \in T x_{n}$ such that $\lambda_{n} \rightarrow \infty$ and

$$
y_{n}^{*}+\lambda_{n} C x_{n}+\varepsilon J x_{n}=\eta_{n} y_{0}^{*} .
$$

Dividing above by $\lambda_{n}$ and taking into consideration that $\left\{y_{n}^{*}+\varepsilon J x_{n}\right\}$ is bounded, we obtain

$$
\lim _{n \rightarrow \infty}\left(C x_{n}-\frac{\eta_{n}}{\lambda_{n}} y_{0}^{*}\right)=0 .
$$

By (109), $\left\|C x_{n}\right\| \geq \alpha$. Thus, (113) implies that the sequence $\left\{\eta_{n} / \lambda_{n}\right\}$ is bounded. We may assume that $\eta_{n} / \lambda_{n} \rightarrow \mu \in(0, \infty)$. Obviously, this implies $\eta_{n} \rightarrow \infty$. Consequently, from (112) we obtain

$$
\frac{\lambda_{n}}{\eta_{n}}\left\|C x_{n}\right\| \rightarrow\left\|y_{0}^{*}\right\|=1
$$

This, along with

says that

$$
\frac{\lambda_{n}}{\eta_{n}} C x_{n} \rightarrow y_{0}^{*},
$$

$$
\frac{C x_{n}}{\left\|C x_{n}\right\|} \rightarrow y_{0}^{*}
$$

which contradicts $\left(110_{a}\right)$. It follows that (111) is true.

Now assume that $(T+\tilde{\lambda} C+\varepsilon J) x \not \supset 0, x \in D(T+C) \cap \partial B_{r}(0)$. Then (111) holds for every $\eta \geq 0$. We claim that the degree $d\left(T+\widetilde{\lambda} C+\varepsilon J, B_{r}(0), 0\right)$, which is well defined by (111), equals 0 . In fact, assume that the contrary holds and consider the homotopy function

$$
H_{n}(t, x) \equiv(T+\widetilde{\lambda} C+\varepsilon J) x-n t y_{0}^{*}, \quad(t, x) \in[0,1] \times\left(D(T+C) \cap \overline{B_{r}(0)}\right) .
$$

We note that this is an admissible homotopy for our degree in 15] (see [15, Theorem 3 , (iv)]). Because of this, we have

$$
d\left(H_{n}(1, \cdot), B_{r}(0), 0\right)=d\left(H_{n}(0, \cdot), B_{r}(0), 0\right)=d\left(T+\lambda C+\varepsilon J, B_{r}(0), 0\right) \neq 0 .
$$

Consequently, for every $n$, the inclusion

$$
T x_{n}+\widetilde{\lambda} C x_{n}+\varepsilon J x_{n} \ni n y_{0}^{*}
$$

is solvable with solution $x_{n} \in B_{r}(0)$. This, however, is a contradiction to the boundedness of the operator $T+\widetilde{\lambda} C+\varepsilon J$. It follows that $d\left(T+\widetilde{\lambda} C+\varepsilon J, B_{r}(0), 0\right)=0$. Since

$$
d\left(T+\varepsilon J, B_{r}(0), 0\right)=1,
$$

by Theorem 3, (i), of [15], there must exist $\lambda_{\varepsilon}>0$ such that $\left(T+\lambda_{\varepsilon} C+\varepsilon J\right) x_{\varepsilon} \ni 0$ for some $x_{\varepsilon} \in \partial B_{r}(0)$.

Let $x_{n} \in \partial B_{r}(0)$ solve

$$
T x_{n}+\lambda_{n} C x_{n}+(1 / n) J x_{n} \ni 0 .
$$

Then, since $T$ and $J$ are bounded and $C$ is bounded below, we cannot have a subsequence of $\left\{\lambda_{n}\right\}$ converging to $\infty$ as $n \rightarrow \infty$. Thus, we may assume that $\lambda_{n} \rightarrow \lambda_{0} \in[0, \infty)$. We may also assume that $x_{n} \rightarrow x_{0} \in \overline{B_{r}(0)}$ and $C x_{n} \rightarrow h^{*}$. If $\lambda_{0}=0$, then the $\left(S_{q}\right)$-property of $T$ implies that $x_{n} \rightarrow x_{0} \in \partial B_{r}(0)$, while its demiclosedness says that $x_{0} \in D(T) \cap \partial B_{r}(0)$ and $T x_{0}=0$, i.e. a contradiction. It follows that $\lambda_{0}>0$. 
We can now work as in the proof of Theorem 8 to show that

$$
\limsup _{n \rightarrow \infty}\left\langle C x_{n}, x_{n}-x_{0}\right\rangle \leq 0 .
$$

This implies that $x_{n} \rightarrow x_{0} \in D(C)$ and $C x_{0}=h^{*}$. Again, the demiclosedness of $T$ says that $x_{0} \in D(T)$ and $T x_{0}+\lambda_{0} C x_{0} \ni 0$. Since $r \geq r_{0}$ is arbitrary, we have that the nonzero eigenvectors of problem (98) form a continuous branch of infinite length.

\section{Applications}

Application 1. This application is connected with Theorem 3. We shall study the existence of eigenvectors of second order nonlinear elliptic equations normalized by their norms in $L^{2}(\Omega)$.

We assume that $\Omega$ is a bounded open set in $\mathcal{R}^{n}$ with boundary $\partial \Omega$ belonging to $C^{2, \alpha}$, for some $\alpha>0$.

Assume that the functions $a_{i}(x, u), i=0,1, \ldots, n$, are defined for $x \in \Omega, u \in \mathcal{R}$, measurable w.r.t. $x$ for all $u$, and continuous w.r.t. $u$ for almost all $x$. We also assume the inequalities

$$
\begin{aligned}
& \left|a_{i}(x, u)\right| \leq \nu_{1}, i=1, \ldots, n, \\
& \left|a_{0}(x, u)\right| \leq \nu_{1}|u|+a(x)
\end{aligned}
$$

with a positive constant $\nu_{1}$ and $a \in L^{2}(\Omega)$.

We consider the eigenvalue problem

$$
\begin{gathered}
\Delta u+\lambda\left\{\sum_{i=1}^{n} a_{i}(x, u) \frac{\partial u}{\partial x_{i}}+a_{0}(x, u)\right\}=0, \quad x \in \Omega, \\
u(x)=0, \quad x \in \partial \Omega,
\end{gathered}
$$

with normalized condition

$$
\|u\|_{L^{2}(\Omega)}=1
$$

We also consider the auxiliary equation

$$
\Delta u+\varepsilon u+\lambda_{\varepsilon}\left\{\sum_{i=1}^{n} a_{i}(x, u) \frac{\partial u}{\partial x_{i}}+a_{0}(x, u)\right\}=0, \quad x \in \Omega .
$$

We study the solvability of the eigenvalue problem (115)-(117) in $W^{2,2}(\Omega) \cap$ $W_{0}^{1,2}(\Omega)$.

Theorem 10. Assume that the above conditions on $\Omega$ and $a_{i}(x, u), i=0,1, \ldots, n$, are satisfied. Assume that for some positive number $\varepsilon_{0}$ and arbitrary $\varepsilon \in\left(0, \varepsilon_{0}\right)$ there exists a number $\lambda_{\varepsilon} \in(0,1]$ such that the problem $((118),(116))$ has no solution $u(x)$ satisfying the conditions

$$
u \in W^{2,2}(\Omega) \cap W_{0}^{1,2}(\Omega), \quad\|u\|_{L^{2}(\Omega)}<1 .
$$

Then there exists a solution $\left(\lambda_{0}, u_{0}\right)$ of the problem (115)-(117) such that $\lambda_{0} \in(0,1]$. 
Proof. We shall apply Theorem 3 with $X=L^{2}(\Omega)$. We define the operators $T, C$ as follows:

$$
\begin{aligned}
T u & =\Delta u, \quad D(T)=W^{2,2}(\Omega) \cap W_{0}^{1,2}(\Omega), \\
C u & =\left\{\sum_{i=1}^{n} a_{i}(x, u) \frac{\partial u}{\partial x_{i}}+a_{0}(x, u)\right\}, \quad \mathcal{D}(C)=W^{1,2}(\Omega) .
\end{aligned}
$$

The solvability of the equation

$$
\Delta u+\tau u=f(x)
$$

in $W^{2,2}(\Omega) \cap W_{0}^{1,2}(\Omega)$ is well known with boundary condition (117), $\tau>0$ and $f \in L^{2}(\Omega)$. Consequently, the operator $T$ is maximal monotone.

Now, we verify conditions $c_{1}$ ),$c_{2}$ ) for the operator $C$. To see that condition $c_{1}$ ) is satisfied, let $u \in D(T)$ and let the inequalities

$$
\langle T u+C u, u\rangle \leq 0, \quad\|u\| \leq S
$$

hold, where $\|\cdot\|$ is the norm in $L^{2}(\Omega)$. The first inequality in (121) implies

$$
\int_{\Omega}\left\{\sum_{i=1}^{n}\left|\frac{\partial u}{\partial x_{i}}\right|^{2}+\left[\sum_{i=1}^{n} a_{i}(x, u) \frac{\partial u}{\partial x_{i}}+a_{0}(x, u)\right] u\right\} d x \leq 0 .
$$

Using the inequalities (114) and the second inequality in (121), we obtain immediately from (122) the estimate

$$
\|u\|_{W^{1,2}(\Omega)} \leq K_{1}(S) .
$$

From (123) we have $\|C u\| \leq K_{2}(S)$ with some number $K_{2}(S)$ depending only on known parameters and $S$. Therefore the quasiboundedness of $C$ with respect to $T$ is established.

To check condition $c_{2}$ ), let $\left\{u_{n}\right\} \subset D(T)$ be such that $u_{n} \rightarrow u_{0}, C u_{n} \rightarrow h_{0}$ and

$$
\limsup _{n \rightarrow \infty}\left\langle C u_{n}, u_{n}-u_{0}\right\rangle \leq 0, \quad\left\langle T u_{n}+C u_{n}, u_{n}\right\rangle \leq 0 .
$$

As in the case of (123), we have from (124)

$$
\left\|u_{n}\right\|_{W^{1,2}(\Omega)} \leq K_{3}
$$

which guarantees that $u_{n} \rightarrow u_{0}, u_{0} \in D(C)$. Using the weak convergence of $\frac{\partial u_{n}}{\partial x_{i}}$ in $L^{2}(\Omega)$ and the strong convergence of $u_{n}(x)$ in $L^{2}(\Omega)$, we obtain $C u_{0}=h_{0}$.

It is easy to show that all the other conditions of Theorem 3 are satisfied. This completes the proof.

Application 2. Consider the elliptic problem

$$
\begin{aligned}
& -(1+\mu) \sum_{i=1}^{n} \frac{\partial}{\partial x_{i}}\left[|\nabla u|^{m-2} \frac{\partial u}{\partial x_{i}}\right]+\frac{c(x)}{|x|^{r}}|u|^{m-1}+\lambda \frac{f(x)}{|x|^{q}}|u|^{m-2} u \\
= & -\sum_{i=1}^{n} \frac{\partial}{\partial x_{i}} h_{i}(x), \quad x \in \Omega,
\end{aligned}
$$

$$
u(x)=0, \quad x \in \partial \Omega,
$$

where $\mu>0, \lambda \geq 1,0<r \frac{m n}{m n-n+m}<q<n, h_{i} \in L^{\frac{m}{m-1}}(\Omega), c, f \in L^{\infty}(\Omega)$, $f(x) \geq 1,1<m<n, \gamma>0$. 
We shall consider solutions $u(x)$ of the problem ((125), (126)) such that

$$
u(x) \in \stackrel{\circ}{W}^{1, m}(\Omega), \quad \frac{1}{|x|^{q}}|u(x)|^{m-1} \in L^{1}(\Omega),
$$

and equation (125) is satisfied in the sense of distributions.

Remark 2. We shall study the problem ((125), (126)) using a variant of Theorem 5 . Namely, we assume conditions $\left.\left.t_{1}\right)-t_{4}\right)$ for the operator $T$ without the restriction $L=D(T)$. The proof of such a variant remains the same.

Theorem 11. Assume that $0 \in \Omega$ and let $\lambda$ be such that the homogeneous problem ((125), (126)) (with $\left.h_{i}(x) \equiv 0\right)$ has only the zero solution for any $\mu<0$. Then the problem $((125),(126))$, for $\mu=0$, has a solution for any functions $h_{i} \in L^{\frac{m}{m-1}}(\Omega)$, $i=1, \ldots, n$.

Proof. We shall apply Theorem 5 with $X=\stackrel{\circ}{W^{1, m}}(\Omega)$ and operators $T, C, \tilde{J}_{m}$ defined as follows:

$$
\begin{aligned}
& \langle T u, \varphi\rangle=\int_{\Omega} \frac{f(x)}{|x|^{q}}|u|^{m-2} u \varphi d x \\
& \langle C u, \varphi\rangle=\int_{\Omega}\left\{\sum_{i=1}^{n}|\nabla u|^{m-2} \frac{\partial u}{\partial x_{i}} \frac{\partial \varphi}{\partial x_{i}}+\frac{c(x)}{|x|^{r}}|u|^{m-1} \varphi\right\} d x, \\
& \left\langle\tilde{J}_{m} u, \varphi\right\rangle=\sum_{i=1}^{n} \int_{\Omega}|\nabla u|^{m-2} \frac{\partial u}{\partial x_{i}} \frac{\partial \varphi}{\partial x_{i}} d x .
\end{aligned}
$$

We let $D(T)$ be the set of all functions $u(x)$ satisfying conditions (127) and the inequality

$$
\left.\left|\int_{\Omega} \frac{f(x)}{|x|^{q}}\right| u\right|^{m-2} u \varphi d x \mid \leq C_{1}\|\varphi\|_{W^{1, m}(\Omega)},
$$

for all $\varphi \in C_{0}^{\infty}(\Omega)$, with some constant $C_{1}$ depending on $u$.

We let $D(C)$ be the set of all functions $u(x) \in \stackrel{\circ}{W^{1, m}}(\Omega)$ satisfying the inequality

$$
\left.\left|\int_{\Omega} \frac{c(x)}{|x|^{r}}\right| u\right|^{m-2} u \varphi d x \mid \leq C_{2}\|\varphi\|_{W^{1, m}(\Omega)}
$$

for $\varphi \in C_{0}^{\infty}(\Omega)$ with $C_{2}$ depending on $u$.

The proof of the needed properties of the operator $T$ was established in the paper [14. It is therefore omitted.

We shall check the properties $\left.c_{1}\right), c_{2}$ ) of the operator $C$.

To show $c_{1}$ ), let us assume that $u \in D(T) \cap D(C)$

$$
\langle T u+C u, u\rangle \leq 0, \quad\|u\| \leq S .
$$

Then we have, immediately,

$$
\int_{\Omega}\left\{|\nabla u|^{m}+\frac{1}{|x|^{q}}|u|^{m}\right\} d x \leq C_{3} .
$$


The estimate for the norm $\|C u\|$ follows from the inequality

$$
\begin{gathered}
\left.\left|\int_{\Omega} \frac{c(x)}{|x|^{r}}\right| u\right|^{m-1}|\varphi| d x \mid \leq C_{4}\left\{\int_{\Omega}\left(\frac{1}{|x|^{r}}|u|^{m-1}\right)^{\frac{m n}{m n-n+m}} d x\right\}^{\frac{m n-n+m}{m n}} \cdot\|\varphi\| \\
\leq C_{5}\left\{\int_{\Omega} \frac{1}{|x|^{q}}|u|^{m} d x\right\}^{\frac{r}{q}} \cdot\left\{\int_{\Omega}|u|^{\frac{m n}{n-m}} d x\right\}^{\left(m-1-m \frac{r}{q}\right) \frac{n-m}{m n}} \cdot\|\varphi\|,
\end{gathered}
$$

and shows that the operator $C$ is quasibounded.

For the proof of $c_{2}$ ), let us consider a sequence $\left\{u_{n}\right\} \subset D(T) \cap D(C)$ such that $u_{n} \rightarrow u_{0}, C u_{n} \rightarrow h_{0}$ and

$$
\limsup _{n \rightarrow \infty}\left\langle C u_{n}, u_{n}-u_{0}\right\rangle \leq 0, \quad\left\langle T u_{n}+C u_{n}, u_{n}\right\rangle \leq S .
$$

Working as in (132), we obtain

$$
\lim _{n \rightarrow \infty} \int_{\Omega} \frac{c(x)}{|x|^{r}}\left|u_{n}\right|^{m-1}\left(u_{n}-u_{0}\right) d x=0,
$$

and the first inequality of (133) implies $u_{n} \rightarrow u_{0}$. It is easy to show that $u_{0} \in$ $\mathcal{D}(C), C u_{0}=h$.

The assertion of Theorem 11 follows now from Theorem 5 and Remark 2.

\section{REFERENCES}

[1] V. Barbu, Nonlinear Semigroups and Differential Equations in Banach Spaces, Noordhoff Int. Publ., Leyden (The Netherlands), 1975. MR0390843 (52:11666)

[2] H. Brézis, M. G. Crandall and A. Pazy, Perturbations of nonlinear maximal monotone sets in Banach spaces, Comm. Pure Appl. Math. 23 (1970), 123-144. MR0257805 (41:2454)

[3] F. E. Browder, Nonlinear Operators and Nonlinear Equations of Evolution in Banach Spaces, Proc. Symp. Pure Appl. Math., 18, Part 2, Providence, 1976. MR0405188 (53:8982)

[4] F. E. Browder, Fixed point theory and nonlinear problems, Bull. Amer. Math. Soc. 9 (1983), 1-39. MR0699315 (84h:58027)

[5] F. E. Browder, The degree of mapping and its generalizations, Contemp. Math. 21 (1983), 15-40. MR 0729503 (85e:47086)

[6] F. E. Browder, Degree of mapping for nonlinear mappings of monotone type, Proc. Nat. Acad. Sci. USA 80 (1983), 1771-1773. MR0699437 (84g:47046)

[7] I. Cioranescu, Geometry of Banach Spaces, Duality Mappings and Nonlinear Problems, Kluwer Acad. Publ., Boston, 1990. MR1079061 (91m:46021)

[8] S. Fučík, J. Nečas, J. Souček and V. Souček, Spectral Analysis of Nonlinear Operators, Lect. Notes Math. \#346, Springer, New York, 1973. MR0467421 (57:7280)

[9] Z. Guan and A. G. Kartsatos, On the eigenvalue problem for perturbations of nonlinear accretive and monotone operators in Banach spaces, Nonlinear Anal. 27 (1996), 125-141. MR.1389473 (97b:47053)

[10] Z. Guan, A. G. Kartsatos and I. V. Skrypnik, Ranges for densely defined generalized pseudomonotone perturbations of maximal monotone operators, J. Differential Equations 188 (2003), 332-351. MR1954518 (2003m:47096)

[11] A. G. Kartsatos, New results in the perturbation theory of maximal monotone and $m$-accretive operators in Banach spaces, Trans. Amer. Math. Soc. 348 (1996), 1663-1707. MR.1357397 (96j:47050)

[12] A. G. Kartsatos and I. V. Skrypnik, Normalized eigenvectors for nonlinear abstract and elliptic operators, J. Differential Equations 155 (1999), 443-475. MR.1698562 (2000f:47101)

[13] A. G. Kartsatos and I. V. Skrypnik, Topological degree theories for densely defined mappings involving operators of type $\left(S_{+}\right)$, Adv. Differential Equations 4 (1999), 413-456. MR.1671257 (2000a:47127) 
[14] A. G. Kartsatos and I. V. Skrypnik, Invariance of domain for perturbations of maximal monotone operators in Banach spaces (to appear).

[15] A. G. Kartsatos and I. V. Skrypnik, A topological degree theory for densely defined quasibounded $\left(\widetilde{S}_{+}\right)$-perturbations of multivalued maximal monotone operators in reflexive Banach spaces, Abstr. Appl. Anal. (to appear).

[16] H.-Z. Li and F.-L. Huang, On the nonlinear eigenvalue problem for perturbations of monotone and accretive operators in Banach spaces, Sichuan Daxue Xuebao (J. Sichuan Univ.) 37 (2000), 303-309. MR1784055 (2001h:47099)

[17] N. G. Lloyd, Degree Theory, Cambridge Univ. Press, Cambridge, 1978. MR0493564 $(58: 12558)$

[18] D. Pascali and S. Sburlan, Nonlinear Mappings of Monotone Type, Sijthoff and Noordhoof, Bucharest, 1978. MR0531036 (80g:47056)

[19] W. V. Petryshyn, Approximation-solvability of Nonlinear Functional and Differential Equations, Marcel Dekker, New York, 1993. MR.1200455 (94f:47081)

[20] E. H. Rothe, Introduction to Various Aspects of Degree Theory in Banach Spaces, Math. Surveys and Monographs, \#23, A.M.S., Providence, 1986. MR0852987 (87m:47145)

[21] J. Simons, Minimax and Monotonicity, Lecture Notes in Mathematics, \#1693, SpringerVerlag, Berlin, 1998. MR 1723737 (2001h:49002)

[22] I. V. Skrypnik, Nonlinear Higher Order Elliptic Equations, Naukova Dumka, Kiev, 1973. MR0435590 (55:8549)

[23] I. V. Skrypnik, Methods for Analysis of Nonlinear Elliptic Boundary Value Problems, Amer. Math. Soc. Transl., Ser. II, \#139, Providence, Rhode Island, 1994. MR.1297765 (95i:35109)

[24] E. Zeidler, Nonlinear Functional Analysis and Its Applications, II/B, Springer-Verlag, New York, 1990. MR1033498(91b:47002)

Department of Mathematics, University of South Florida, Tampa, Florida 33620-5700

E-mail address: hermes@math.usf.edu

Institute for Applied Mathematics and Mechanics, R. Luxemburg Str. 74, Donetsk 340114, UKRAINE

E-mail address: skrypnik@iamm.ac.donetsk.ua 